SAND94-0048

Unlimited Release

Printed January 1994
Distribution

UC-700

\title{
Hydrodynamics of Maneuvering Bodies: LDRD Final Report
}

\author{
S. N. Kempka \\ Computational Fluid Dynamics Department \\ and \\ J. H. Strickland \\ Unsteady Aerodynamics Department \\ Sandia National Laboratories \\ Albuquerque, NM 87185
}

\begin{abstract}
The objective of the "Hydrodynamics of Maneuvering Bodies" LDRD project was to develop a Lagrangian, vorticity-based numerical simulation of the fluid dynamics associated with a maneuvering submarine. Three major tasks were completed. First, a vortex model to simulate the wake behind a maneuvering submarine was completed, assuming the flow to be inviscid and of constant density. Several simulations were performed for a dive maneuver, each requiring less than $20 \mathrm{cpu}$ seconds on a workstation. The technical details of the model and the simulations are described in a separate document, but are reviewed herein. Second, a gridless method to simulate diffusion processes was developed that has significant advantages over previous Lagrangian diffusion models. In this model, viscous diffusion of vorticity is represented by moving vortices at a diffusion velocity, and expanding the vortices as specified by the kinematics for a compressible velocity field. This work has also been documented previously, and is only reviewed herein. The third major task completed was the development of a vortex model to describe inviscid internal wave phenomena, and is the focus of this document. Internal wave phenomena in the stratified ocean can affect an evolving wake, and thus must be considered for naval applications. The vortex model for internal wave phenomena includes a new formulation for the generation of vorticity due to fluid density variations, and a vortex adaption algorithm that allows solutions to be carried to much longer times than previous investigations. Since many practical problems require long-time solutions, this new adaption algorithm is a significant step toward making vortex methods applicable to practical problems. Several simulations are described and compared with previous results to validate and show the advantages of the new model. An overview of this project is also included.
\end{abstract}

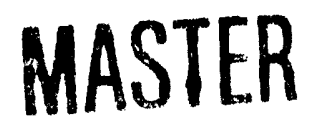




\section{Executive Summary}

Several issues associated with the evolution of submarine wakes are of considerable interest to the naval community. Two areas of interest are the detection of others' submarines, and stealth operation of our own. Accordingly, there is a strong interest in developing numerical simulation capabilities to gain a better understanding of the complex hydrodynamic evolution of submarine wakes. With this motivation, and with the naval community in mind as a potential customer, a gridless, vorticity-based model was developed as part of the LDRD project entitled "The Hydrodynamics of Maneuvering Bodies" (FY 92 and 93).

The reason that a vorticity-based method was proposed and implemented is that vortex methods offer the possibility of performing the simulations of interest where conventional numerical methods (e.g., finite element or finite difference methods) are not feasible. Our success in developing such a model strongly suggests that vortex methods hold promise in this area. A further indication of the promise of vortex methods is that we are now aware that a naval research laboratory is exploring this approach with university faculty.

Vortex methods are significantly less well-developed than conventional finite element and finite difference methods. Thus, there is a wide range of areas that need to be addressed in the development of vortex methods. The naval community has focused much of its efforts on agglomeration methods and implemention of vortex methods on massively parallel computers. Our focus is both more applied and more fundamental, and complementary to the naval effort. It is more applied in the sense that we implemented a model to describe the evolution of the wake behind an actual submarine during a realistic maneuver [21], which to the best of our knowledge has not been accomplished elsewhere. By necessity, several simplifying assumptions were used in the model. Notably, the vorticity generation as a result of density variations in the ocean (variations in salinity and temperature) was omitted, as were the effects of fluid viscosity. Encouraged by the short execution times of the simplified model (less than 20 seconds on a Sun SPARC workstation), more fundamental efforts were undertaken to include vorticity generation and viscous effects for inclusion in the submarine model.

As a result of those efforts, a new viscous diffusion model, referred to as the non-solenoidal diffusion velocity method, was developed. This work is described in [14], has been presented at a recent American Physical Society meeting, and has been submitted to a refereed technical journal. Unlike previous gridless diffusion methods, the non-solenoidal diffusion velocity method can actually be used to solve diffusion equations, not just to add a secondary diffusive effect to flow fields. Thus, the non-solenoidal diffusion velocity method can be applied in a wide variety of other applications in which mass diffusion is readily described, in addition to diffusion of vorticity.

A new formulation to include vorticity generation was also developed, including a new vortex adaption algorithm that conserves circulation, ensures monotonicity, and preserves symmetry. The vorticity generation model and vortex adaption algorithm are described in detail in this report. 
Although we have not yet obtained funding from the naval community (largely as a result of decreasing naval budgets), the accomplishments of this LDRD have already had significant impact beyond meeting the goals of the LDRD. As a direct result of our accomplishments, vortex methods are now more applicable to practical problems than ever before. Accordingly, we will host a workshop (Feb. 1995) on the use of vortex methods in engineering problems. Invited participants will include staff from academia, industry, and other national laboratories.

Additionally, new research programs have been initiated to further advance vortex methods beyond existing fluid dynamics capabilities. One area of interest is in coating flows, such as those used to manufacture photographic film, porous membranes, special coatings for glass, and the filling of casting molds. A common characteristic of these flows is that complex free surface phenomena are important to obtaining a defect-free product. The gridless nature of vortex methods is a natural approach to treat free surface phenomena (as discussed in this report), and thus vortex methods are being developed for these applications. Another area of interest is the simulation of combustion processes. Toward this end, we have established contacts with staff at the Combustion Research Facility that have a long history of outstanding contributions in vortex dynamics. Additionally, it appears that ongoing work in the area of pool fire simulation and smoke transport could benefit significantly from the advances made in this LDRD project. Thus, this LDRD has not only provided capabilities for the intended naval community, but is the basis for several new research and application programs. 


\section{Acknowledgments}

We thank our supervisors, Steve Rottler, 1511, and Chris Hailey, 1552, for their support and advice in the completion of this project, and in their support for the continued activities that have arisen from this work.

We also thank Micheal Glass, 1511 for writing the software used to visualize the vortex method simulations. He wrote the $\mathrm{C}++$ functions used to write EXODUS II data files, the AVS modules that read the EXODUS II files, the code to generate "filled" objects, and numerous other graphics capabilities that were indispensable. Additionally, the versatility and ease of use of these graphics capabilities allowed a new approach to writing software, in which the graphics capabilities are used to detect errors without ever having to look at ASCII data files of numbers. This procedure is referred to as "graphical debugging," and allows "bugs" to be found much more quickly than by inspection of numbers, thus reducing the overall time to develop software.

Lastly, we thank James Schutt, 1511, for providing a great deal of guidance in programming the numerical algorithms discussed in this report in the $\mathrm{C}++$ computer language. He also provided preliminary versions of $\mathrm{C}++$ class libraries being developed as part of the ongoing LDRD project entitled "An Object-Oriented Finite Element Code Architecture for Massively Parallel Computers." These libraries significantly simplified the "bookkeeping" in the code, especially in the implementation of the time-integrator. 
Table of Contents

Hydrodynamics of Maneuvering Submarines: LDRD Project Overview ......................8

1. Objective and Motivation for this LDRD Project ..................................................8

2. Chronology of Developments ........................................................................9

3. Summary of Simulation of the Wake Behind a Maneuvering Submarine ...........10

4. Lagrangian Simulation of Viscous Diffusion ......................................................11

Simulation of Incompressible Flows with Variations in Fluid Density ...........................14

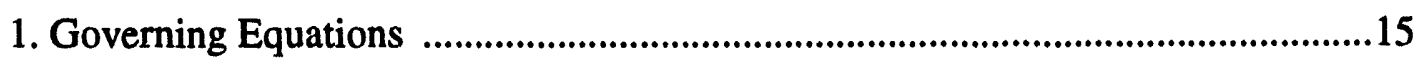

2. Lagrangian Vortex Model Formulation .............................................................18

2.1 Discrete Representation of Vorticity Fields ............................................20

2.2 The Vortex Method for Baroclinic Flows ..................................................26

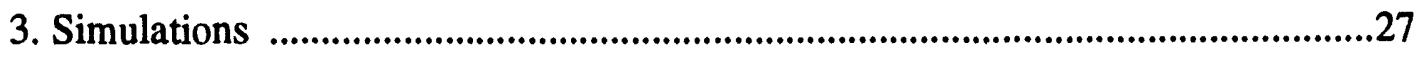

4. Summary ….............................................................................................................37

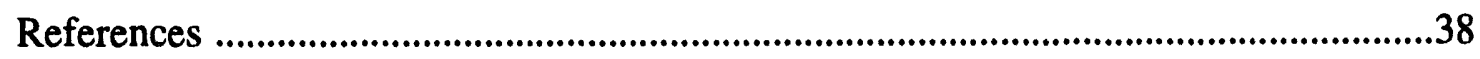




\section{Hydrodynamics of Maneuvering Submarines: LDRD Project Overview}

The objective of this section is to provide an overall perspective on the three technical accomplishments of this LDRD project. The majority of this report focuses on only one of these accomplishments: the development of an algorithm to simulate baroclinic vorticity generation (which results from variations in fluid density), including a vortex adaption algorithm that greatly extends the allowable simulation times. The other two accomplishments of this LDRD project have already been documented in detail. They are the simulation of the wake behind a maneuvering submarine [21], and a gridless method to simulate diffusion processes [14]. Thus, an overall description of the project is lacking, and is therefore described in this introductory section before proceeding to the technical description of the baroclinic vorticity algorithm.

\section{Objective and Motivation for this LDRD Project}

The objective of this LDRD project was to use a Lagrangian, vorticity-based method to simulate the complex flow associated with a maneuvering submarine. Vorticity-based methods are particularly well-suited to large scale flows since only rotational regions need to be resolved. A familiar example is that of a typical boundary layer flow. Vorticity occurs only in the boundary layer itself, thus only the boundary layer need be resolved. The irrotational outer region, which occupies most of the grid in velocity-pressure formulations, need not be discretized in vorticity-based methods. In problems such as the flow around a submarine, where the boundary layer thickness is very small compared to the submarine length (thus requiring many grid points in grid-based methods), the reduced grid requirements associated with vorticity-based methods are particularly beneficial.

Similarly, the length scales of the vorticity shed from maneuvering submarines are small compared to the length over which they persist. A vortex trailing from a diving plane might be a few feet in diameter, whereas the trail of vortices might persist over miles. In this case, Lagrangian vortex methods provide an even greater advantage: the vorticity of interest can be tracked without a grid. Thus, Lagrangian vortex methods have two significant advantages over velocity-pressure based formulations. First, fewer grid points are needed to resolve the flow in terms of its vorticity, and second, a Lagrangian vortex model requires no grid.

Vorticity-based methods, have received relatively little development effort compared to finite element and finite difference methods for velocity-pressure formulations of the Navier-Stokes equations. Thus, a significant effort was made in this LDRD to not only develop a capability to predict the wake behind a maneuvering submarine, but also to contribute to the advancement of vorticity-based methods. Advances were made in three areas: 1) a fast-executing model was developed for simulating the evolution of the wake behind a maneuvering submarine, 2) a Lagrangian formulation was developed that includes the effects of fluid density variations, including an adaption scheme to maintain high resolution and high accuracy of Lagrangian methods, and 3) a Lagrangian technique was developed for simulating viscous diffusion of vorticity. 
The chronological order of these efforts does not ccincide with the order in which they have been documented. To avoid confusion that might arise because of this, the chronology is briefly described.

\section{Chronology of Developments}

The wake evolution model and simulations for several submarine maneuvers were completed first, and are der a ed in a Sandia Report [21]. Next, the new method to include density variation effects was formulated and implemented. As discussed below, a new vortex adaption technique had to be developed in order to maintain solution accuracy.

The development of a new adaption technique was unanticipated (and hence, not part of the original proposal) since there was evidence in the literature that existing adaption techniques were adequate. We found, however, that the previously developed techniques introduced non-physical features into the solution. Apparently, the non-physical features did not manifest themselves for the relatively short times considered in previous analyses, but became important for the longer times of interest to us.

The new adaption scheme resolved the aforementioned loss of accuracy at long simulation times, but as a result of providing more accurate solutions at later times, an additional unexpected phenomenon occurred. A conclusion of several previous investigations (e.g., Anderson [1], Meng [15]) was that the overall configuration of vorticity did not depend strongly on the vortex size. However, the previous investigations did not have the benefit of our adaption model which allows longer simulation times and higher resolution. We found that, contrary to the results of the previous investigations, the solution depends strongly on the size of the vortices at simulation times beyond those of the previous investigations. The phenomenon we observed is that, as the vortex size was reduced from one simulation to another, additional small-scale features arose in the flow field. Similar observations were recently reported by Rottman and Stansby [20]. At the large simulation times of interest, these small-scale features can have a significant effect on the overall configuration of vorticity.

This phenomenon is believed to be a feature of the inviscid equations of motion. As noted by Dritchel [9], "the nearly inevitable and incessant drive of an inviscid fluid to produce finer and finer scales of motions prevents any finite algorithm from accurately modeling even the largest scales of motion for arbitrarily long time." In addition, fluid interfaces in inviscid vortical flows are believed to achieve infinite curvatures in finite time [20]. In a real, viscous fluid, viscous diffusion eliminates all scales that are smaller than a certain finite length. The smallest length scale that exists (i.e., is not eliminated by viscous diffusion) depends on the relative magnitudes of inertial and viscous terms in the NavierStokes equations, as indicated by the Reynolds number. As the Reynolds number increases, the effect of viscosity decreases relative to inertia, and smaller and smaller length scales are generated, ending in what is referred to as turbulence.

This brings to mind a comparison of Lagrangian vortex methods and Eulerian pressurevelocity methods. Eulerian methods require "numerical viscosity" for numerical stability. 
Lagrangian vortex methods do not require numerical viscosity, and thus provide a more accurate solution to the inviscid equations. But, as mentioned above, the inviscid equations generate infinitesimally small scales. This is not apparent in simulations using Eulerian methods, since the numerical viscosity required for stability artificially eliminates most small scales. Often, the viscosity required for numerical stability is larger than the actual fluid viscosity [17], casting doubt on the accuracy of the simulation.

Lagrangian vortex methods, on the other hand, attempt to resolve all the small scales since viscosity is not required for numerical stability. They fail to do so, however, only as a result of the finiteness of numerical spatial resolution (finite spacing between finite vortices). However, viscous effects can be simulated more accurately since artificial viscosity does not influence real viscous effects.

In the flows of interest, simulation times were such that there was sufficient time for relatively small length scales to be generated (it takes a finite time for the production of small scales to commence). Thus, viscous diffusion had to be included in the Lagrangian diffusion model for this project.

Numerous methods to include viscous diffusion exist (e.g., Chorin [7], Russo [19], Cottet [8], Fishelov [10]), however, each approach has significant deficiencies that are untenable for the simulations of interest. A recent approach by Ogami and Akamatsu [16] was particularly appealing, so we implemented it. However, we quickly found it to be highly inaccurate. Nonetheless we believed that Ogami and Akamatsu's basic idea was a good one, and thus embarked on an investigation to find the reason that it was inaccurate. As it turned out, a relatively simple modification to their proposed method made it highly accurate, but the time taken to develop the new diffusion model was essentially the remainder of the time allotted to the LDRD project.

Thus, to summarize the history of this project, efforts to include vorticity generation by variations in fluid density required the development of a new adaption algorithm and a new method to simulate diffusion in a Lagrangian model. The development of the diffusion model is believed to be a significant technical development in the fluid dynamics simulation community, and thus its development was documented in a Sandia Report [14], submitted to the Journal of Computational Physics, and presented at the 1993 American Physical Society Division of Fluid Dynamics meeting. The initial simulations of the inviscid, constant density evolution of the wake behind a maneuvering submarine were documented earlier, as they were completed [21]. These previously documented results are reviewed briefly in the following sections. Thereafter, the technical details are presented for the previously undocumented work regarding the new formulation to describe vorticity generation by fluid density variations, including a new vortex adaption algorithm.

\section{Summary of Simulation of the Wake Behind a Maneuvering Submarine}

We performed several vortex simulations of the evolution of the vortices trailing from the dive planes on a diving submarine, as described in [21]. As mentioned earlier, the naval 
community has an intense interest in the evolution of the wake behind submarines for purposes of detecting other submerged submarines and in keeping our own submarines undetected. A principal feature of interest is the upward motion of the vortices trailing from the dive planes of a diving submarine. The fluid dynamics of interest are essentially embodied in Newton's law that for every action there is an equal and opposite reaction. In the case of a diving submarine, the reaction to the force pushing the submarine downward is the upward motion of the trailing vortices.

The model described in [21] addresses this mechanism, and was used to simulate the wake evolution associated with several different dive maneuvers of a full-scale submarine. Significant differences occurred in the wake evolutions for the different maneuvers, and were readily interpreted in terms of maneuvers to avoid so as to avoid detection, and features to watch for when searching for other submarines.

A few details of this model are that a multi-degree-of-freedom dynamics code is used to predict the hydrodynamic forces on the submarine for a particular trajectory. The strength of the vortices shed from the dive planes is determined from well-known relationships between hydrodynamic forces and the vorticity on control surfaces, including hull vortices. The paths of these vortices are then tracked using a Lagrangian vortex method.

These types of simulations would not even be considered if a grid-based, velocity-pressure Navier-Stokes simulation had to be used. The reason for this is that the number of grid points required would be prohibitively large. Typically, the submarine traveled several thousand feet $\left(\mathrm{O}\left(10^{3}\right) \mathrm{ft}\right.$ ) over the course of the simulation, and the wake typically traveled several hundred feet $\left(\mathrm{O}\left(10^{2}\right) \mathrm{ft}\right)$ in the vertical direction. The diameter of trailing vortices is typically on the order of a foot. In order to resolve vortices of this size, a grid spacing of $1 / 10$ foot would be needed, or $\left(\mathrm{O}(10)\right.$ points per $\mathrm{ft}$, or $\left(\mathrm{O}\left(10^{3}\right)\right.$ points per $\left.\left.\mathrm{ft}^{3}\right)\right)$. The number of grid points would be $\mathrm{O}\left(10^{9}\right)$ : $\left(\mathrm{O}\left(10^{3}\right)\right.$ grid points $\left./ \mathrm{ft}^{3}\right) \cdot\left(\mathrm{O}\left(10^{3}\right) \mathrm{ft}\right.$ of travel distance) $\left(\mathrm{O}\left(10^{2}\right) \mathrm{ft}\right.$ (vertical)) $\left(\mathrm{O}\left(10^{l}\right) \mathrm{ft}\right.$ (horizontal)).

This huge number of grid points could be reduced by a few orders of magnitude (at least) by judicious gridding methods, and perhaps, adaptive grid techniques. Nonetheless, there would still be far too many grid points to consider performing the simulation. On the other hand, the simulations described in [21] required less than 20 seconds of execution time on a SPARC workstation. These simulations are not as detailed as typical velocity-pressure Navier-Stokes simulation, but they demonstrate that they provide a tool for use in engineering analyses where no other practical tools are available.

\section{Lagrangian Simulation of Viscous Diffusion}

A method to simulate viscous diffusion of vorticity in a Lagrangian reference frame has long been sought. Without a method to simulate viscous diffusion, vortex methods are essentially inviscid, which greatly limits their applicability. Moreover, there is controversy as to whether there is "grid-convergence" of solutions to the inviscid equations for rotational flows, since the equations appear to generate infinitesimally small length-scales that cannot be resolved numerically. Viscosity in real fluids attenuates the smallest length 
scales, thus allowing convergence of numerical solutions when the grid spacing is on the order of the smallest length scale of the flow.

A large number of algorithms to simulate Lagrangian diffusion have been developed previously, which itself is a testament to the demand for such a model. The principal difficulty is representing the spatial derivatives in the Laplacian of the vorticity field $\nabla^{2} \omega$ without the benefit of a grid. A brief list of previous algorithms begins with the Gaussian random walk method by Chorin [7], which exploits the isomorphic relationship between diffusion and Gaussian probability density distribution. However, this method is applicable only for large Reynolds numbers, and converges slowly ("statistical convergence"). Cottet and Mas-Gallic [8] include diffusion by convolving the velocity of point vortices with the fundamental solution to the diffusion equation,

$$
\frac{1}{4 \pi v t} e^{-\frac{r^{2}}{4 \pi v t}}
$$

where $t$ is time, $r$ is the distance from the vortex center and $v$ is the viscosity. In this method, vorticity diffuses from one vortex to another. At the edge of a vorticity field, diffusion into regions where there was initially no vorticity requires that new, zero circulation vortices be added to act as receptacles for the diffusive flux into irrotational regions. This leads to a proliferation of vortices, which is undesireable. However, if receptacle vortices are not added, the method does not conserve circulation [18]. In addition, the use of point vortices limits the practical application of this method.

Fishelov [10] approximates the Laplacian of the vorticity field by differentiating the core function of each vortex, and then summing the contributions of each vortex to the Laplacian at each point. This allows the viscous flux from one vortex to another to be calculated, which also requires the addition of receptacle vortices in order to conserve circulation, as in the method of Cottet and Mas-Gallic. Russo [19] describes a method in which a Voronoi grid is determined at each time step depending on the locations of vortices. The grid readily allows the Laplacian of the vorticity field to be approximated. This method is stable and conserves circulation, but the computation to generate the grid at each time step appears to be computationally expensive. We also note that the commonly-used diffusing core algorithm has been shown to be incorrect [11].

To conclude this brief (and non-exhaustive) list of diffusion methods, the diffusion velocity algorithm by Ogami and Akamatsu [16] is described. In the diffusion velocity method, a diffusive flux of vorticity, $-v \nabla \omega$, is represented as a convective flux of the local vorticity, $\vec{u}_{d} \omega$, where the diffusion velocity $\vec{u}_{d}$ is determined from the relation $\vec{u}_{d} \omega=-v \nabla \omega$. The vorticity and its gradient are evaluated using the method by Fishelov [10]. Each vortex is then convected at the local diffusion velocity to simulate diffusion.

This method has the desirable characteristic that receptacle vortices are not needed, since there is no exchange of vorticity from one vortex to another. As a result, circulation is conserved identically. However, upon implementing this method, we found that it yielded accurate results only if the vortices were highly overlapped. For example, if the distance 
between the centers of adjacent vortices is only one-tenth of the core radius (so the vortices are highly overlapped), reasonable results can be obtained only for short times, $v t / \sigma^{2}<1,(v$ is the viscosity, $t$ is time, and $\sigma$ is the core size). For longer times, the value of the vorticity at the core centers is too large, and non-physical wiggles develop in the regions between vortices. These errors arise even more quickly if the distance between adjacent vortices is a single core radius, which is a much more reasonable spacing in terms of computational feasibility.

Careful examination of the diffusion velocity method showed that the errors occur because two important aspecis of the problem were being omitted. First, the diffusion velocity is non-solenoidal: $\nabla \bullet \vec{u}_{d} \neq 0$. Second, the mathematics of the governing equations require that, if the circulation of each vortex remains constant as it convects at the diffusion velocity, then the size of each core must change size according to the local divergence of the diffusion velocity (see [14] for details). This change of core size is specified by the kinematics of the flow field, and can be implemented by solving an additional ordinary differential equation. Incorporating the expansion of vortices yields the results that no wiggles arise in the solution field, and that the solution remains accurate for very long times. Apparently, as vortices become separated, the cores expand just the right amount to keep the cores overlapped and, hence, keep the solution smooth. Without core expansion, the solution rapidly looses smoothness and accuracy as the cores become separated.

We refer to our new technique as the non-solenoidal diffusion velocity method. This method can be easily incorporated into existing vortex methods. Additionally, the non-solenoidal diffusion velocity method guarantees that circulation is conserved since the circulation of each vortex never changes. Lastly, the non-solenoidal diffusion velocity method satisfies both the dynamics (the diffusion equation) and the kinematics (details associated with the time-differentiation of the circulation in a reference frame moving at the diffusion velocity).

This method also has far-reaching implications for the simulation of mass diffusion. In particular, a density field can be represented in terms of small regions of mass with a specified density distribution, just as a vorticity field is represented in terms of vortices, which are nothing more than small regions of circulation with a specified vorticity distribution. In this way, flows involving both mass diffusion and viscous diffusion can be considered, such as the coating flows described in the Executive Summary. 


\section{Simulation of Incompressible Flows with Variations in Fluid Density}

We begin the description of the formulation to simulate flow fields in which motion arises as a result of spatial variations in fluid density in a gravitational field. This topic is of interest since submarine wakes can be affected by the combination of gravity and the stratification of the ocean. Recall that the ocean is a stably stratified fluid consisting of layers of fluid with different densities due to variations of temperature and salinity. The stratification is referred to as being stable since, upon being disturbed, the density field will eventually return to its initial state. The physical mechanism by which the fluid returns to its initial state is the phenomena commonly referred to as internal waves, which have the well-known Brunt-Väisälä frequency scale. Internal waves are essentially the result of vorticity which is generated whenever the density gradient is misaligned with the pressure gradient, which is referred to as baroclinic vorticity generation. The flow induced by the baroclinically-generated vorticity returns the fluid to its equilibrium state (in which the density and pressure gradients are aligned).

We note that the flow being discussed is incompressible even though the fluid density is not constant. This is due to the fact that the volume of a fluid element does not change with time, which is the kinematic definition of incompressibility. Even as the volume of a fluid element remains constant, its density can change as a result of mass diffusion. A significant difference between constant density flows and variable density, incompressible flows is the manner by which vorticity is created. In constant density flows, the only sources of vorticity are interactions of a viscous fluid with a boundary. In the interior of such flows, viscosity neither creates nor destroys vorticity, it only re-distributes existing vorticity [2]. As mentioned above, in variable density flows, vorticity can be created in the interior of the fluid. Thus, incompressible flows with non-constant density are fundamentally different from constant density flows.

Three advances were made in the development of a Lagrangian vortex model to simulate baroclinic flows. First, a new adaption technique was developed to maintain the resolution of the vorticity field. Second, a new formulation of the baroclinic vorticity generation term in the vorticity equation was derived which does not require that the density gradients be approximated for fluids in which the density differences are small compared to the average fluid density. Lastly, convergence characteristics of transient solutions with respect to the size of vortices were addressed. This last topic has received scant attention in the literature, with most of the attention being devoted to a different type of convergence. The type of convergence usually discussed relates to the use of vortices to provide a discrete representation of a smooth vorticity field. Convergence in this sense, which has been proven by Hald [13], means that as the size of the vortices decreases (keeping their relative overlap constant), errors (the difference between the true vorticity field and its discrete representation) decrease. These issues are very important to the development and acceptance of vortex methods as useful tools for practical problems. 
As an overview of this section, the following topics are described:

- the governing equations and vortex method basics are presented, including a description of the method used to calculate the velocity field from the vorticity field,

- a new formulation for the baroclinic vorticity generation term is described,

- discrete representations of vorticity fields are discussed, as related to the need for adaption and the new adaption technique,

- several simulations of a specific flow field are presented and compared with previous simulations, and,

- the issue of convergence of transient solutions for different core sizes is discussed.

\section{Governing Equations}

The flow of interest is assumed to be inviscid and incompressible. Although the simulations to be shown are two-dimensional, the formulation is developed for three-dimensional flow, the only exception tising that the calculation of the baroclinic term is formulated for only two-dimensional flow. Mass diffusion is assumed to be negligible, so that if the interface between regions of different density $\rho$ is initially sharp, it remains sharp for all time. Conservation of mass of the fluid is given by

$$
\frac{\partial \rho}{\partial t}+(\vec{u} \bullet \nabla) \rho=0
$$

where the velocity field is $\vec{u}$. The momentum equation is

$$
\rho\left[\frac{\partial \vec{u}}{\partial t}+(\vec{u} \bullet \nabla) \vec{u}\right]=-\nabla P+\rho \vec{g} .
$$

The fluid pressure is $P$ and the body acceleration is $\vec{g}$, which for this discussion is assumed to be the downward acceleration due to gravity. Since the variations of fluid density in the ocean are small compared to the average fluid density, the well-known Boussinesq approximation can be used, in which the density is approximated as $\rho(\vec{x})=\rho_{0}+\rho^{\prime}(\vec{x})$, where $\rho_{0}$ is a constant reference density, and $\rho^{\prime}$ is a local deviation from the average. Substituting $\rho=\rho_{0}+\rho^{\prime}$, into Eq. (2),

$$
\left(\rho_{\circ}+\rho^{\prime}\right) \frac{D \vec{u}}{D t}=-\nabla P+\rho_{\circ} \vec{g}+\rho^{\prime} \vec{g} .
$$

Applying the assumption of a small density variations, $\left|\rho^{\prime}\right|$ « $\rho_{o}$, to only the left-hand side of this equation yields the approximate Boussinesq momentum equation,

$$
\rho_{\circ} \frac{D \vec{u}}{D t}=-\nabla P+\rho_{\circ} \vec{g}+\rho^{\prime} \vec{g} .
$$


Rather than solving the above equation, we wish to solve its vorticity form, since it has a very simple Lagrangian interpretation which will be discussed in the next section. To obtain the vorticity form of the Euler equation, the curl operator is applied to the entire equation, and using the definition of vorticity, $\vec{\omega}=\nabla \times \vec{u}$, yields

$$
\frac{D \vec{\omega}}{D t}=(\vec{\omega} \bullet \nabla) \vec{u}+\nabla \times\left(\rho^{\prime} \vec{g}\right) / \rho_{0} .
$$

The curl of the terms $-\nabla P+\bar{\rho} \vec{g}$ is zero since the curl of the gradient of a scalar $(P)$ is zero identically, as is the curl of the constant gravity vector. Noting that for a vector $\delta \vec{\delta} l$ with infinitesimal length, on which every point moves at the local fluid velocity, the time evolution of $\vec{\delta} l$ is [2],

$$
\frac{d \vec{\delta} l}{d t}=(\vec{\delta} l \bullet \nabla) \vec{u}
$$

Since this is the same form as Eq. (5), except for the last term in Eq. (5), the physical interpretation of Eq. (5) is that the vorticity vector evolves as a fluid vector with a source term which is proportional to $\nabla \times\left(\rho^{\prime} \vec{g}\right) / \bar{\rho}_{\text {. }}$.

If we had not approximated the density variations, the right-hand side of the above equation would be $-\nabla \times(\nabla P / \rho)=\nabla \rho \times \nabla P / \rho^{2}$. A physical interpretation of how the cross product of the density and pressure gradients can create vorticity is shown in Figure 1 . We note that, with regard to baroclinic vorticity generation, the small-density variation approximation is equivalent to approximating the pressure gradient as being hydrostatic; i.e., $\nabla P=\rho \vec{g}$.

The benefit of the small density variation approximation is that the baroclinic generation of vorticity can be evaluated without having to know the pressure. Only knowledge of the density field, which is known, is required to evaluate $\nabla \times\left(\rho^{\prime} \vec{g}\right) / \bar{\rho}$. Tryggvason [22] shows that the small density variation approximation is equivalent to the limit of a large Froude number and a small Atwood number.

The only remaining issue is how to determine the velocity field for use in the vorticity equation. In principle, the velocity field is determined from the coupled equations,

$$
\nabla \bullet \vec{u}=0 \quad \text { and } \quad \nabla \times \vec{u}=\vec{\omega}
$$

where it is seen that the velocity is essentially determined by the inverse operation of the curl operator, subject to the constraint that the velocity field is solenoidal. A general solution to this problem, with velocity boundary conditions $\vec{u}_{b}$, was derived in [24] as, 


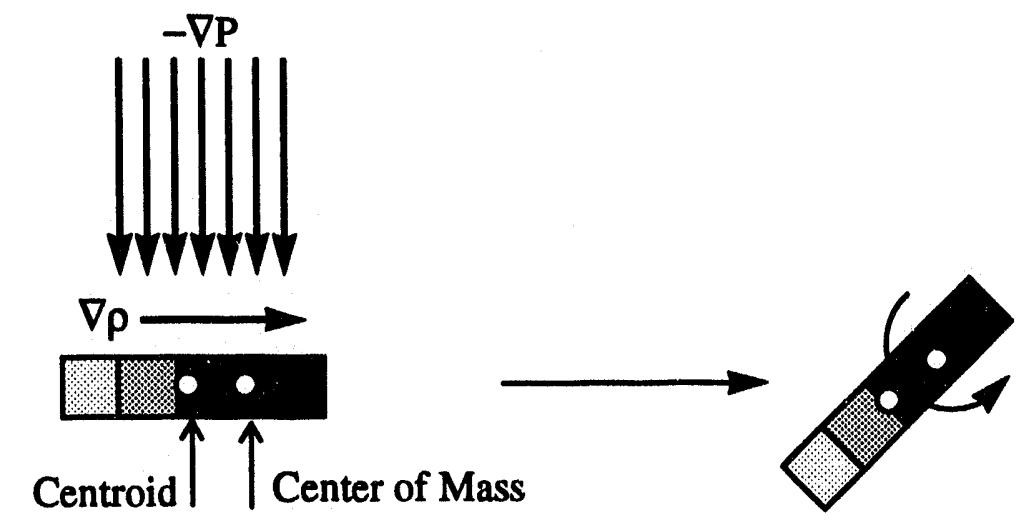

Figure 1. Generation of vorticity when pressure gradients $\nabla P$ and density gradients $\nabla \rho$ are not aligned. A fluid element is shown which has a density gradient along its longest dimension, as indicated by the shading, where darker shading indicates a higher density. If a pressure gradient is applied uniformly as shown, the displacement of the less dense fluid will be greater, owing to its smaller moment of inertia. This type of motion involves a rotation of the fluid element about its center of mass, as shown. From another point of view, consider that the center of mass is offset from the centroid by some distance in the direction of the density gradient, as shown. Also recall that pressure acts through the centroid, but the fluid element rotates about the center of mass. Thus, a torque is generated on the fluid element if the pressure gradient is not aligned with the line between the centroid and the center of mass. In either case, the fluid element rotates, indicating that vorticity is generated.

$$
\begin{gathered}
\vec{u}(\vec{x}) \beta_{d}=\int_{V} \frac{\vec{\omega}\left(\vec{x}^{\prime}\right) \times\left(\vec{x}-\vec{x}^{\prime}\right)}{\left|\vec{x}-\vec{x}^{\prime}\right|^{d}} d V\left(\vec{x}^{\prime}\right) \\
\int_{S} \frac{\left[\vec{u}_{b}\left(\vec{x}_{b}{ }^{\prime}\right) \bullet \hat{n}\left(\vec{x}_{b}\right)\right]\left(\vec{x}-\vec{x}_{b}{ }^{\prime}\right)+\hat{n}\left(\vec{x}_{b}{ }^{\prime}\right) \times \vec{u}_{b}\left(\vec{x}_{b}{ }^{\prime}\right) \times\left(\vec{x}-\vec{x}_{b}{ }^{\prime}\right)}{\left|\vec{x}-\vec{x}^{\prime}\right|^{d}} d S\left(\vec{x}_{b}{ }^{\prime}\right)
\end{gathered}
$$

where $\mathrm{V}$ is the volume of the domain, $\mathrm{S}$ is the boundary of the domain, locations on the boundary $S$ are denoted as $\vec{x}_{b}$, and the unit normal vector on the boundary is $\hat{n}$. The coefficient $\beta_{d}$ is $2 \pi$ for two dimensions $(d=2)$, and $4 \pi$ for three dimensions $(d=3)$. The two terms of the integrand of the boundary integral represent the influence of the normal velocity boundary condition and the tangential velocity boundary condition, respectively.

Eq. (6) allows the velocity field to be calculated from a vorticity field and velocity boundary conditions, but the user must be warned that arbitrary vorticity fields and velocity boundary conditions do not, in general, comprise a well-posed mathematical problem. For example, Stokes theorem shows that there is a relationship between a vorticity field and the tangential velocity boundary condition, 


$$
\int_{A} \vec{\omega} \bullet \hat{n} d A=\oint \vec{u}_{b} \bullet d \vec{c}
$$

One interpretation of this relationship, for two-dimensions, is that the area integral of the vorticity must equal the line integral of the tangential velocity on the boundary. For example, if the tangential velocity is zero on the entire boundary, then the area integral of the vorticity field must be zero. If Stokes' theorem is not satisfied, then the problem is not well-posed.

The area integral of the vorticity is referred to as the circulation, and is used extensively in the formulation of Lagrangian vortex methods, as discussed next.

\section{Lagrangian Vortex Model Formulation}

The basis for Lagrangian vortex methods was first described by Helmholtz in 1858. Helmholtz' most important finding was that inviscid vorticity transport is equivalent to the transport of a fluid vector with infinitesimal length, as alluded to previously. This remarkable finding can be derived by considering a differential fluid volume $\delta V$ that is a truncated cylinder with cross-sectional area $d \vec{A}$ and outward point normal unit vector $\hat{n}$, and differential length $\delta \dot{l}$, for which $\delta V=\delta \vec{A} \bullet \delta \dot{l}$. Every point in the volume is assumed to convect at the local fluid velocity, i.e.,

$$
\frac{d \vec{x}}{d t}=\vec{u}(\vec{x})
$$

Kinematical considerations (see [2]) indicate that the time-rate-of-change of the cross-sectional area element $\delta \vec{A}$ is, in indicial notation,

$$
\frac{d \delta A_{i}}{d t}=\delta A_{i} \frac{\partial u_{j}}{\partial x_{j}}-\delta A_{j} \frac{\partial u_{j}}{\partial x_{i}}
$$

These relations are used in the time-differentiation of the circulation as in the following equations. 


$$
\begin{gathered}
\frac{d}{d t} \int_{A} \vec{\omega} \bullet \hat{n} d A=\int_{A}\left(\frac{\partial \omega_{i}}{\partial t}+\frac{d x_{j} \partial \omega_{i}}{d t} \frac{\partial x_{j}}{\partial x_{i}} d A+\int_{A} \omega_{i} \frac{d\left(d A_{i}\right)}{d t}\right. \\
=\int_{A}\left(\frac{\partial \omega_{i}}{\partial t}+u_{j} \frac{\partial \omega_{i}}{\partial x_{j}}\right) n_{i} d A+\int_{A} \omega_{i}\left(\frac{\partial u_{j}}{\partial x_{j}} d A_{i}-\frac{\partial u_{j}}{\partial x_{i}} d A_{j}\right) \\
=\int_{A}\left(\frac{\partial \vec{\omega}}{\partial t}+(\vec{u} \bullet \nabla) \vec{\omega}\right) \bullet \hat{n} d A+\int_{A}[\vec{\omega}(\nabla \bullet \vec{u})-(\vec{u} \bullet \nabla) \vec{\omega}] \bullet \hat{n} d A \\
=\int_{A}\left[\frac{\partial \vec{\omega}}{\partial t}+(\vec{u} \bullet \nabla) \vec{\omega}+\vec{\omega}(\nabla \bullet \vec{u})-(\vec{u} \bullet \nabla) \vec{\omega}\right] \bullet \hat{n} d A
\end{gathered}
$$

For incompressible flows, $\nabla \bullet \vec{u}=0$, so that

$$
\frac{d}{d t} \int_{A} \vec{\omega} \bullet \hat{n} d A=\int_{A}\left[\frac{\partial \vec{\omega}}{\partial t}+(\vec{u} \bullet \nabla) \vec{\omega} \cdots(\vec{u} \bullet \nabla) \vec{\omega}\right] \bullet \hat{n} d A .
$$

Substituting the vorticity-form of the Euler equations Eq. (5) into Eq. (11) yields

$$
\frac{d}{d t} \int_{A} \vec{\omega} \bullet \hat{n} d A=\int_{A}\left[\nabla \times\left(\rho^{\prime} \vec{g}\right) / \rho_{0}\right] \bullet \hat{n} d A
$$

This equation indicates that as a region of vorticity convects at the local fluid velocity, the time-rate-of-change of circulation is given by the area integral of the baroclinic source term. In practice, this means that the Euler equations are satisfied if vortices are convected at the local fluid velocity while the circulation changes according to the area integral of the baroclinic source term. This is the principal basis for vortex methods. In previous methods (e.g., [1]), however, the curl operation in the baroclinic term is performed as

$$
\nabla \times\left(\rho^{\prime} \vec{g}\right) / \rho_{0}=\frac{\nabla \rho^{\prime} \times \vec{g}}{\rho_{\circ}} .
$$

This expression requires a representation for $\nabla \rho^{\prime}$, which typically requires some type of approximation; e.g., $\nabla \rho^{\prime}\left(\vec{x}_{i}\right) \approx\left(\rho^{\prime}\left(\vec{x}_{i+1}\right)-\rho^{\prime}\left(\vec{x}_{i-1}\right)\right) /\left(\vec{x}_{i+1}-\vec{x}_{i-1}\right)$. Models that use this approach have been developed previously. However, this approximation is often inaccurate, especially if the density fields have a very small diffusivity, as in the case of interest.

A formulation that depends only on $\rho^{\prime}$ itself can be obtained by applying Stokes' theorem Eq. (7) to the right-hand side of Eq. (12) to yield 


$$
\frac{d}{d t} \int_{A} \vec{\omega} \bullet \hat{n} d A=\oint\left(\rho^{\prime} \vec{g}\right) / \rho_{o} \bullet d \vec{c} .
$$

This equation allows the time-rate-of-change of circulation to be calculated by performing a line integral, which generally can be performed more accurately than approximating a derivative, then integrating over an area, as required in the usual formulation. Moreover, this new formulation allows the simple interpretation that circulation is generated whenever the integration path has a component parallel to the gravitational vector. Details of how to implement this formulation depend on how the vorticity field is discretized, which is discussed next.

\subsection{Discrete Representation of Vorticity Fields}

Two important aspects of implementing a Lagrangian vortex method are: 1) smooth vorticity fields must be accurately represented using finite regions of vorticity, and 2) the representation of the vorticity must remain accurate for long times.

The most common discretization of vorticity fields was proposed by Chorin [7]. He proposed that finite regions of vorticity be assumed to have a circular shape and to have a radially symmetric distribution of vorticity, such as shown in Figure 2 . Chorin called these regions vortex "blobs," and the name has endured. The entire blob is assumed to convect at the velocity of the blob center, and the blob does not change shape as it convects, nor does the shape of the vorticity distribution change. This is an approximation since a region of fluid subjected to shear will, in fact, change shape. Adaption methods can deal with this matter, as discussed below, and are more desirable than having to evaluate the velocity at every point in the field.

The core radius for blobs must be specifed, which is analogous to grid spacing in finite element and finite difference methods. Similarly, a vorticity distribution must be specified for each blob, which is analogous to interpolation functions used in finite difference methods or basis functions in finite element methods.

Chorin accurately simulated flow around a cylinder using this approach, but did not evaluate the accuracy of the blob assumption. Subsequently, several investigators showed that the blob approach is rigorously justifiable. Hald [13] proved that the trajectories of the blobs represent the exact trajectories of particles as the number of blobs increases. Beale and Majda $[3,4]$ then showed that vortex blobs are convergent in the sense that decreasingly small perturbations in the locations of vortex blobs (which represent perturbations in the vorticity field) lead to decreasingly small perturbations in the velocity field.

Before proceeding, a brief description is presented to show how vortex blobs are used to represent a vorticity field, and how the vorticity field is used to obtain the velocity field. Consider the representation of a circular vortex sheet that forms between fluids of different density. A detailed view of this representation is shown in Figure 3. We seek to represent the sheet as having a vorticity value of unity everywhere on the sheet. Each blob is assumed to have a core size $\sigma$ of approximately $0.1 R$, where $R$ is the radius of the circle. 


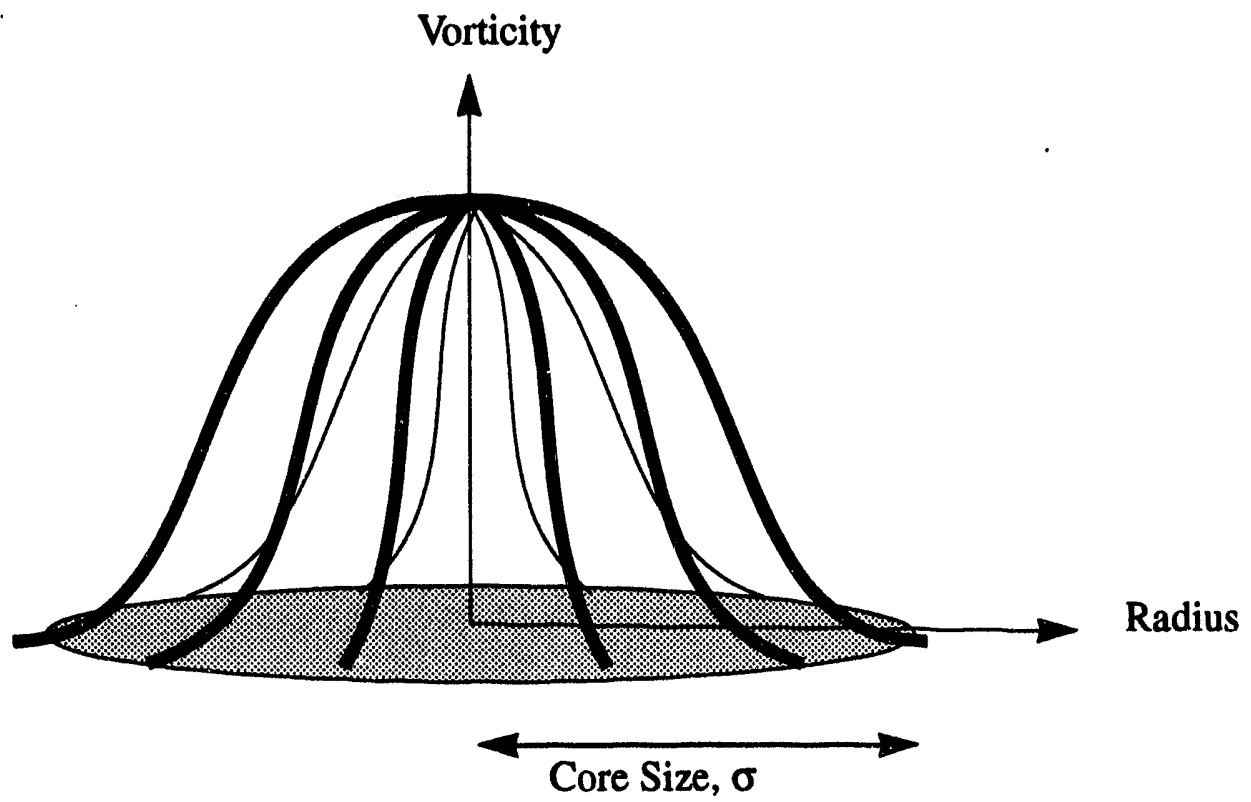

Figure 2 Radially symmetric vorticity distribution assumed for a vortex blob of core radius $\sigma$.

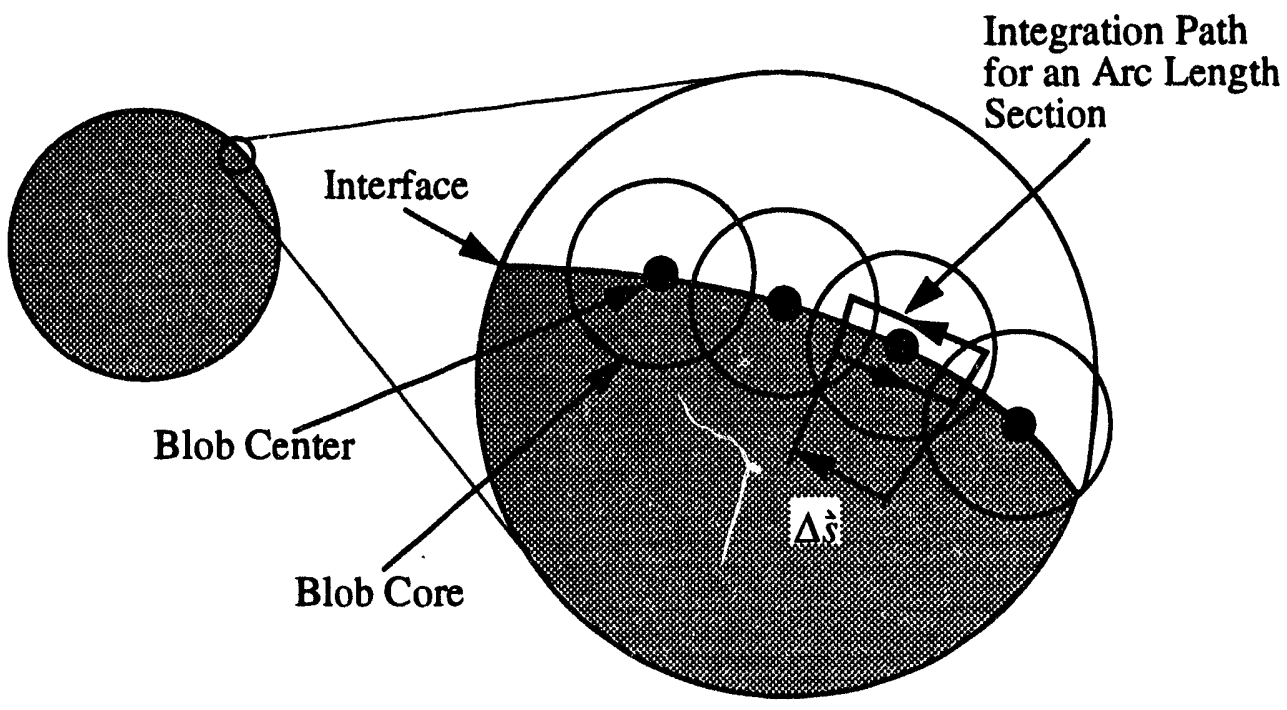

Figure 3 Vortex blob representation of the vortex sheet on the interface between two fluids: a circular region of one fluid in an infinite domain of another fluid. 
This core size is taken to provide reasonable resolution with respect to the size of the circle. Each blob is also assumed to have a Gaussian vorticity distribution,

$$
\vec{\omega}_{i}(\vec{x}, t)=\frac{\Gamma_{i}(t)}{\pi \sigma^{2}} e^{-\left(\frac{\left|\vec{x}-\vec{x}_{i}\right|}{\sigma}\right)^{2}}
$$

where the subscript $i$ denotes a particular vortex, $\vec{x}_{i}$ is the cencer of the $i$-th vortex, and $\Gamma_{i}$ is the circulation of the $i$-th vortex. The actual vorticity field is obtained by superposing the Gaussian fields of the individual vortices,

$$
\vec{\omega}(\vec{x}, t)=\sum_{i=1}^{N} \frac{\Gamma_{i}(t)}{\pi \sigma^{2}} e^{-\left(\frac{\left|\vec{x}-\vec{x}_{i}\right|}{\sigma}\right)^{2}} .
$$

The important feature is that the accuracy of the representation of any vorticity field is determined by how the individual vorticity distributions of blobs overlap one another. For the desired representation of a circular vortex sheet with vorticity of unity, Figure 4 shows the vorticity distribution on a circular arc between blob centers as a function of the distance between blob centers. If the cores are not sufficiently overlapped, then the vorticity field between blobs contains "wiggles" that differ from the desired value of unity. Ghoniem, et al. [12] indicate that if the ratio of the distance between blob centers to that of the core size is less than unity, then at least second order accuracy in the vorticity field is obtained.

A significant problem in vortex methods is to keep the vortices overlapped throughout the duration of a simulation. The principal reason that this difficulty arises is that, as mentioned previously, the vortex cores are assumed to remain circular, when in fact, the flow can distort them to a non-circular shape ${ }^{1}$. For example, cores that deform to become elliptical in shape could remain overlapped, whereas circular cores would become separated, resulting in non-physical wiggles. An example of this is described in [14].

Several approaches have been attempted. Anderson [1] used an initial configuration of vortices that were overlapped to such a large extent that the cores remain overlapped for a considerable time. However, at some time, the cores become separated, thus, the initial configuration determines the allowable simulation time, which is not desirable. Schemes to adapt the vortex field so as to maintain accuracy for extended periods of time have also been developed previously (e.g., Meng [15]), but they do not preserve symmetry when it exists. The basic idea of these previous adaption schemes is that new vortices are inserted between vortices which have become separated. Each new vortex is given $1 / 3$ of the circulation of the adjacent vortices. For example, consider two "parent" vortices that have equal circulations of unity, and have become separated too far to maintain accuracy. Adaption consists of inserting a new "child" vortex between the two "parent" vortices. Each

1. The reason that cores are assumed to remain circular is that it is much more difficult to calculate the velocity field of a non-circular vortex than a circular vortex. 

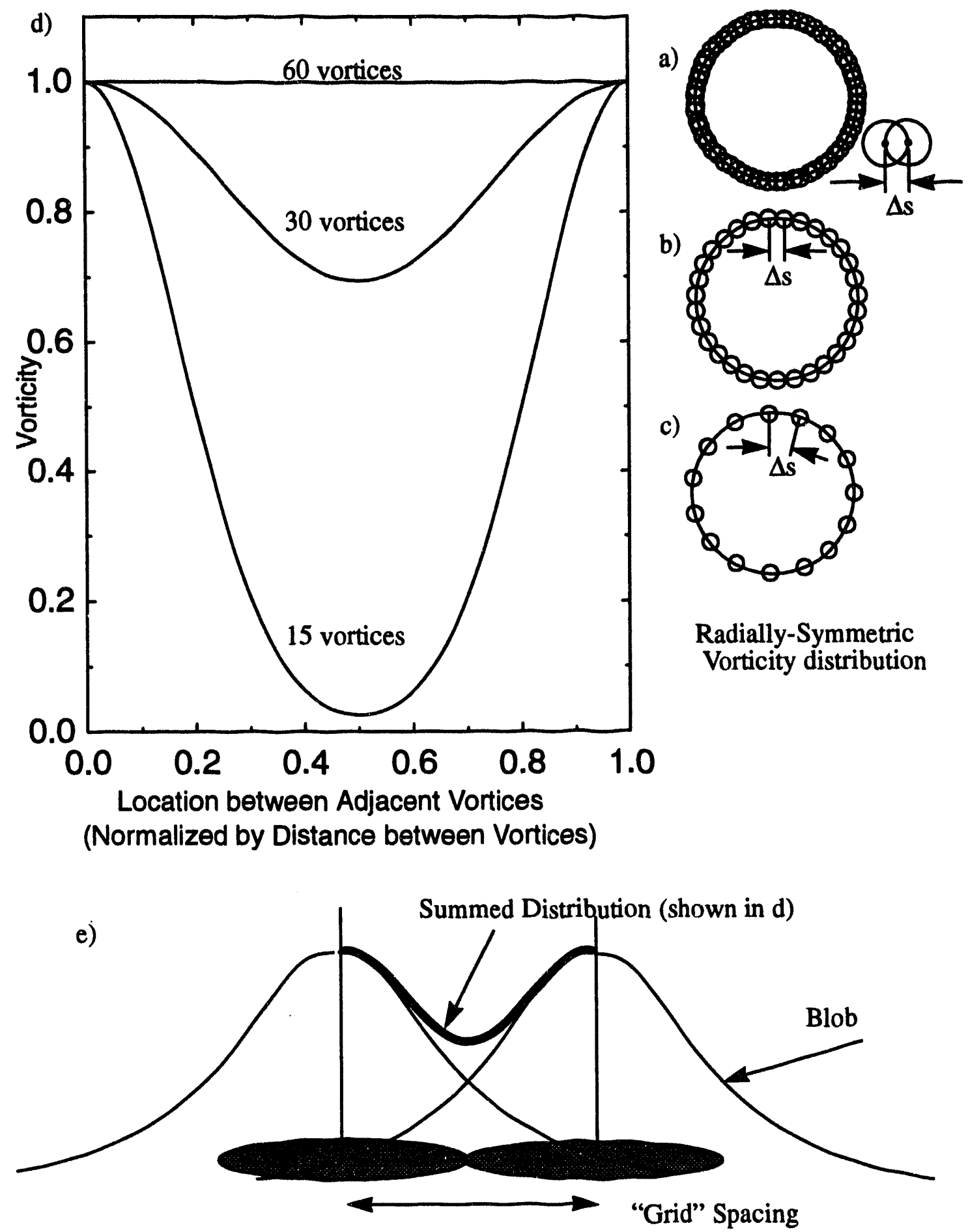

Figure 4 Discrete representations of a circular vortex sheet with uniform vorticity using collections of discrete vortices: a) 60 vortices, b) 30, c) 15 . The main figure d) shows the continuous vorticity field in the regions between vortex centers for the three different numbers of vortices. Since the desired vorticity distribution is unity everywhere on the interface, only the 60 vortex configuration is acceptable. Figure (e) shows a close-up view of the 30 vortex configuration, in which the variation in vorticity between the vortex centers is due to lack of core overlap. 
"parent" contributes a circulation of 1/3 to the "child," so that the "parents" and "child" each have a circulation of $2 / 3$. This algorithm assures conservation of circulation and a region of uniform circulation remains uniform. However, this algorithm does not maintain a region of uniform circulation if contiguous intervals require adaption. Similarly, the simple adaption algorithm does not preserve symmetry, as shown in Figure 5.

To address these issues, we have developed a new adaption algorithm that is monotonic and preserves symmetry. In the new adaption algorithm, contigucus intervals that require adaption are considered as a coupled set, rather than separately as in the simple adaption scheme. As demonstrated in Figure 5, treating one interval at a time leads to loss of symmetry (when it exists). It is assumed that each "parent" vortex in a contiguous interval that requires adaption will contribute the same fraction $f$ of its circulation $\Gamma_{i}$ to new "child" vortices, and the "child" vortices will have circulations $\Gamma_{c, i}$ equal to the arithmetic mean of its adjacent "parent" vortices. In equation form, the new circulation values of parent vortices is

$$
\Gamma_{i}^{\prime}=f \Gamma_{i}
$$

and the circulation of "child" vortices is

$$
\Gamma_{c, i}=\frac{1}{2}\left(\Gamma_{i}^{\prime}+\Gamma_{i+1}^{\prime}\right)
$$

where the $i$-th child lies at the midpoint between the $i$-th and $i+1$-th "parents." Requiring that the total amount of circulation on the interval remain unchanged yields a value for $f$

$$
f=\frac{\sum_{i=1}^{M} \Gamma_{i}}{\sum_{i=1}^{M} \Gamma_{i}+\frac{1}{2} \sum_{i=1}^{M-1}\left(\Gamma_{i}+\Gamma_{i+1}\right)}
$$

where $M$ is the number of vortices on a contiguous interval to be adapted. When the interval is symmetric in circulation, the denominator equals zero. In this case, only half the interval need be considered.

A limitation of this adaption scheme is associated with the assumption that each original vortex contributes the same fraction of its circulation to the new vortices. Essentially, the stretching on each interval is assumed to be uniform. Thus, the vortex field should be checked for adaption often so that the locally uniform stretching assumption is appropriate. As indicated by the simplicity of the algorithm, it is computationally inexpensive, so we typically adapt the field (if necessary) at each time step. Further details of the adaption process, such as how the number of vortices increases with time are described below. 

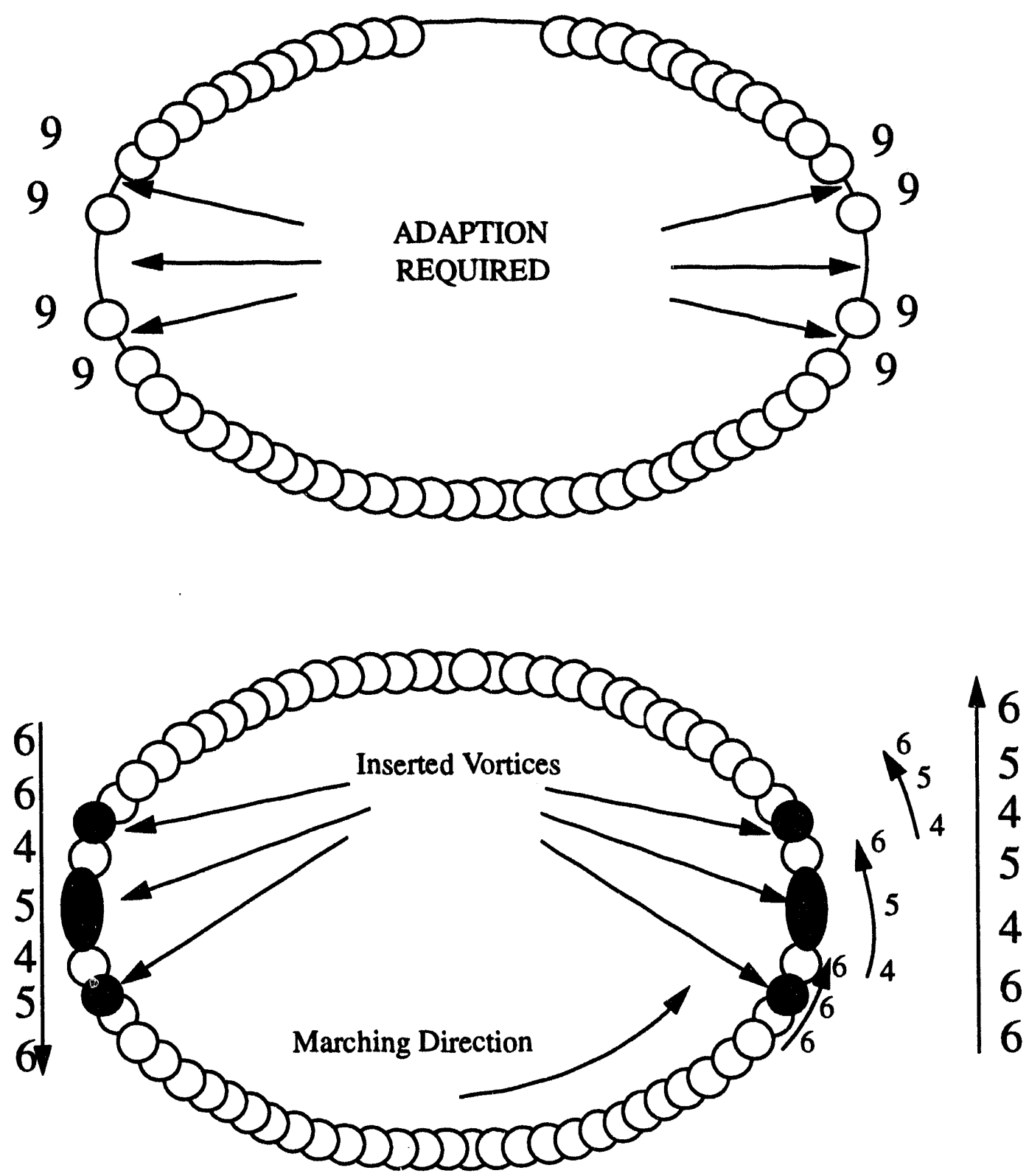

Figure 5 Example of how a simple adaption scheme is non-monotonic and does not preserve symmetry. Intervals between vortices are considered separately, and initially, each vortex on an interval to be adapted has a circulation of 9. Upon adaption, each existing vortex contributes $1 / 3$ of its circulation to the newly inserted vortex. So, on the first interval, the circulations are 6,6,6. The circulations from subsequent adaption are shown to result in nonuniform distributions of circulation (nonmonotonic), and the initial symmetry is lost. 
To summarize this section, it was shown that the spacing between adjacent vortices must be less than or equal the core radii of the vortices in order to avoid non-physical representations of a vorticity field. In order to maintain this overlap as the field evolves, a new monotonic, symmetry preserving, circulation conserving adaption scheme was developed. This adaption scheme allows essentially unlimited simulation times and thus, allows the examination of previously unexplored nonlinear flow regimes, as described next.

\subsection{The Vortex Method for Baroclinic Flows}

The previous considerations were used to formulate a vortex model to describe the motion arising from an unstable configuration of two fluids ${ }^{2}$ with different, non-diffusing densities in a gravity field. Viscous diffusion of vorticity could be included using the non-solenoidal diffusion velocity method (we are presently pursuing this), but the focus of this formulation is the baroclinic generation of vorticity. Thus, each fluid is assumed to be inviscid.

An essential feature of this type of flow is that the only location where non-zero vorticity exists is on the interface between the two fluids. This is because the only locations where vorticity is generated is at locations where the density gradient is non-zero, all of which lie on the interface between the two fluids.

Another important feature of this type of flow is that, for the assumptions in effect, the vorticity remains on the interface between the two fluids for all time. This simplification occurs since, in the absence of mass diffusion and viscous diffusion, the density fields and the vorticity field all convect at the local fluid velocity. Thus, keeping tracking of the vorticity implicitly specifies the location of the density fields. Accordingly, the density difference across each vortex is always the same. This allows the effects of density fields to be known without explicitly solving the conservation of mass equation.

An example of a discrete vortex representation of the interface between two fluids is shown in Figure 3. The interface is discretized into arc lengths $\Delta \vec{s}$, and a vortex exists at the center of each arc interval. Circulation is generated on each interval, and is endowed to the vortex lying on that interval (even though a vortex can extend beyond the bounds of an arc length interval, which always occurs as a result of the necessary condition that adjacent vortices overlap one another).

A simulation consists of calculating the motion of each vortex due to the velocity induced by all the other vortices, and the time evolution of the circulation of each vortex due to baroclinic generation of vorticity. The motion of the $i$-th vortex is

2. The vortex method described here is not restricted to the consideration of only two fluds. In principle, as many fluids as desired can be considered, although complex intersections of several fluids would present considerable difficulty since the density difference at the intersection point of several fluids is not well-defined. Such a situation might be more readily described by representing each region of different density as a collection of blobs that have a mass and a density distribution. The density gradient could then be calculated using the method developed by Fishelov [10], i.e., differentiate the core function of each blob, and superpose them to represent the derivative. This is the approach used to calculate vorticity derivatives in the non-solenoidal diffusion velocity method. 


$$
\frac{d \vec{x}_{i}}{d t}=\vec{u}\left(\vec{x}_{i}\right) .
$$

The velocity at an arbitrary point (including the vortex locations) is obtained from Eq. (6) and the vorticity distribution specified in Eq. (15) (with zero velocity boundary conditions at infinity for simplicity),

$$
\vec{u}(\vec{x})=\frac{1}{2 \pi} \sum_{i=1}^{N} \frac{\Gamma_{i}(t)}{\left|\vec{x}-\vec{x}_{i}\right|}\left[1-e^{-\left(\frac{\left|\vec{x}-\hat{x}_{i}\right|}{\sigma_{i}}\right)^{2}}\right] .
$$

The time-rate-of-change of the circulation for each vortex is,

$$
\frac{d \Gamma_{i}}{d t}=\left[\left(\rho^{\prime}\right)^{+}-\left(\rho^{\prime}\right)^{-}\right] \vec{g} \bullet \Delta \vec{s} / \rho_{o}
$$

where $\Delta \vec{s}$ is the previously mentioned discrete arc length along the interface. This expression is an approximation to the closed line integral around $\Delta \vec{s}$, with the value of $\rho^{\prime}$ being $\rho^{1+}$ on one side of the interface, and $\rho^{\prime-}$ on the other side, the sign depending on the chosen direction of the integration path. Since the interface is assumed to have zero thickness, there are no contributions from the integration paths that are perpendicular to the interface (see Figure 3).

With regard to evaluating Eq. (22), the density difference across the interface is constant, so the only portion of the right-hand side that varies with time is the orientation of the local arc length $\Delta \vec{s}$, with respect to the gravitational vector, as indicated by the vector dot product of $\Delta \vec{s}$ and $\vec{g}$.

To non-dimensionalize these equations, we use the characteristic length scale, $R$, of the disturbance to the density fields to define the dimensionless quantities,

$$
\dot{x}^{*}=\grave{x} / R \quad \Delta \vec{s}^{*}=\Delta \vec{s} / R .
$$

The density variation is non-dimensionalized using the reference density, $\left(\rho^{\prime}\right)^{*}=\rho^{\prime} / \rho_{o}$, and the dimensionless gravity vector is $\vec{g}=\vec{g} /|\vec{g}|$. Using these definitions in Eq. (22) allows the dimensionless time to be defined as $t=t(R /|\vec{g}|)^{-1 / 2}$. The dimensionless velocity is then $\vec{u}=\vec{u}(|\vec{g}| R)^{-1 / 2}$.

\section{Simulations}

The evolution of an initially circular (two-dimensional) region of lower density fluid within an infinite domain of higher density fluid is considered (see Figure 6). Recalling that the objective of this LDRD work was to develop models for submarine wakes and their interaction with the ocean surface, it may not be clear how the motion of a bubble is relevant. 


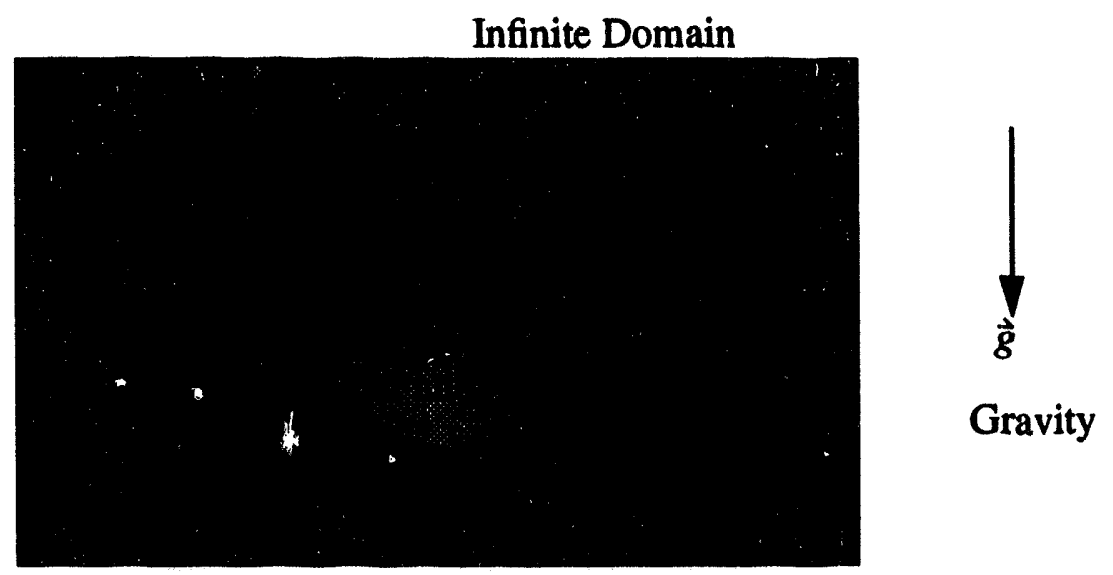

Figure 6 Two-dimensional, circular bubble of fluid within an infinite domain of higher density fluid.

The relevance is that baroclinic vorticity generation is important to both types of flows. For example, as the trailing vortices from a diving submarine convect upward, they disturb the stable stratification of the ocean. That is, the density field is perturbed, and vorticity is generated baroclinically to return the fluid to its equilibrium state. The baroclinically-generated vorticity will also reduce the upward motion of the trailing vortices. The evolution of a bubble depends entirely on baroclinic vorticity generation. Thus, if the bubble flow can be simulated accurately, then the numerical model of baroclinic vorticity generation can be applied to the naval applications of interest. Further, byconsidering bubbles, there is an opportunity to compare the model with previous investigations of bubble phenomena, (e.g., Collins [6] and Anderson [1]).

Returning to the description of bubble flow, the two fluids are assumed to be non-diffusive and inviscid, as mentioned previously. Additionally, surface tension effects are also assumed to be negligible ${ }^{3}$. The reference density for the problem at hand is taken to be the density of the fluid at infinity, so that the dimensionless density perturbation for the heavier fluid is $\rho^{\prime *}=0$. For the lower density fluid in the bubble, $\rho^{\prime^{*}}=0.1$ is specified. The radius of the initial circle is specified as $R=1 \mathrm{~m}$, and the dimensionless core radius of each vortex is specified as $\sigma^{*}=0.1$ for the simulations. Some smaller cores are considered and will be ncied as needed. Gravity is assumed to be in the downward direction $\vec{g}=-\hat{j}$, and $|\vec{g}|=10 \mathrm{~m} / \mathrm{s}^{2}$. These are the same specifications used by Anderson [1], thus allowing a comparison of the present, new formulation with a previous result.

Our numerical simulation of the bubble evolution is shown in Figure 7 in which the "cap" structure becomes apparent at the later times shown. To the best of our knowledge, this structure has not been resolved in previous numerical simulations. Two notable geometric

3. Surface tension effects can also be implemented in vortex methods. Some previous efforts in this area are by Tryggvasson [23] and Zufiria [25]. A new formulation for surface tension by Brackbill, et al. [5] appears to provide an opportunity for a greatly improved approach wherein surface tension can be readily incorporated as a source of circulation at an interface. 


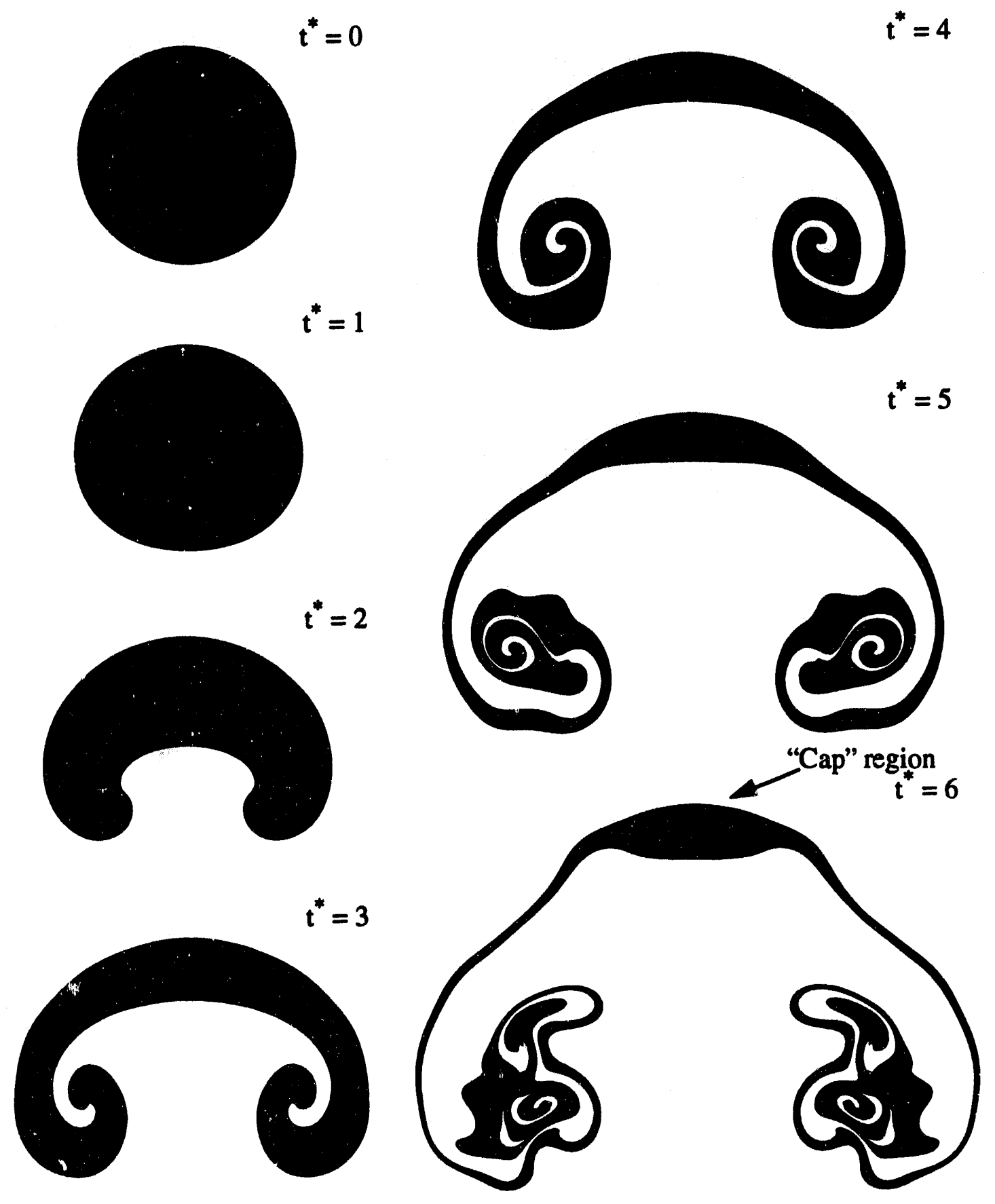

Figure 7 Numerical simulation of the evolution of a bubble of lower density fluid in an infinite region of higher density fluid. The formation of the "cap" at later times is evident. Our new vortex adaption technique allowed this calculation to be carried out to later times that were unaccessible to previous investigations. The radius of the initial interface is 1.0 , and the core radii are 0.1 . 


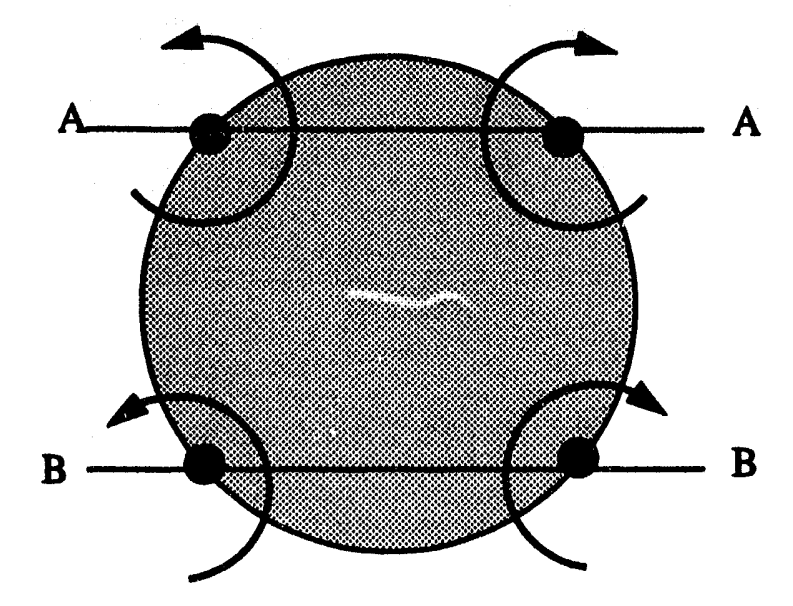

Figure 8 The mechanism for loss of top-bottom symmetry on an initially circular bubble. The vorticity at 90-degree intervals is equal in magnitude but different in direction, as indicated by the rotation directions. The interface region above $\mathrm{A}-\mathrm{A}$ is pushed upward and radially outward, whereas the interface below B-B is pushed upward and radially inward, which destroys the initial symmetry of the system, and leads to further nonlinearities.

features of the bubble at late-times are the "cap" at the top of the bubble, and the highly mixed region beneath the "cap." These features were also observed in an experiment by Collins [6] to investigate the evolution of a two-rimensional bubble using a Hele-Shawtype apparatus.

The solution shown in Figure 7 was calcilated using the new adaption technique. The adaption was specified to maintain $\sigma / \Delta s<2.5$ where $\Delta s$ is the spacing between adjacent cores, and $\sigma$ is the core radius. Recall from the earlier examination of vortex spacing, that good resolution of a field requires that $\sigma / \Delta s>1$. Thus, the use of $\sigma / \Delta s<2.5$ as an adaption criterion should yield a very smooth solution.

A natural question is, why does the bubble interface becomes non-circular? Eq. (22) shows that circulation is generated whenever the interface tangent vector has a vertical component (since gravity is vertical). Thus, since the tangent vector of the interface is nonuniform, the vorticity generation on the interface is nonuniform along the bubble interface. This nonuniformity results in the immediate loss of circular symmetry (see Figure 8) and also is the basis for the nonlinear aspects of the bubble evolution.

Another interesting aspect of the bubble evolution is the increase in the length of the interface with time. As shown in Figure 9, the interface length is initially $2 \pi$, and increases by almost a factor of ten in an elapsed dimensionless time of 7 . The transient length for adaption criteria of $\sigma / \Delta s=1.0,1.5,2.0$, and 2.5 are also shown in order to illustrate the effect of vortex spacing. The number of vortices changes with time in each of these calculations, as shown in Figure 10. As the interface length increases, more vortices are needed in order to maintain the specified core overlap. 


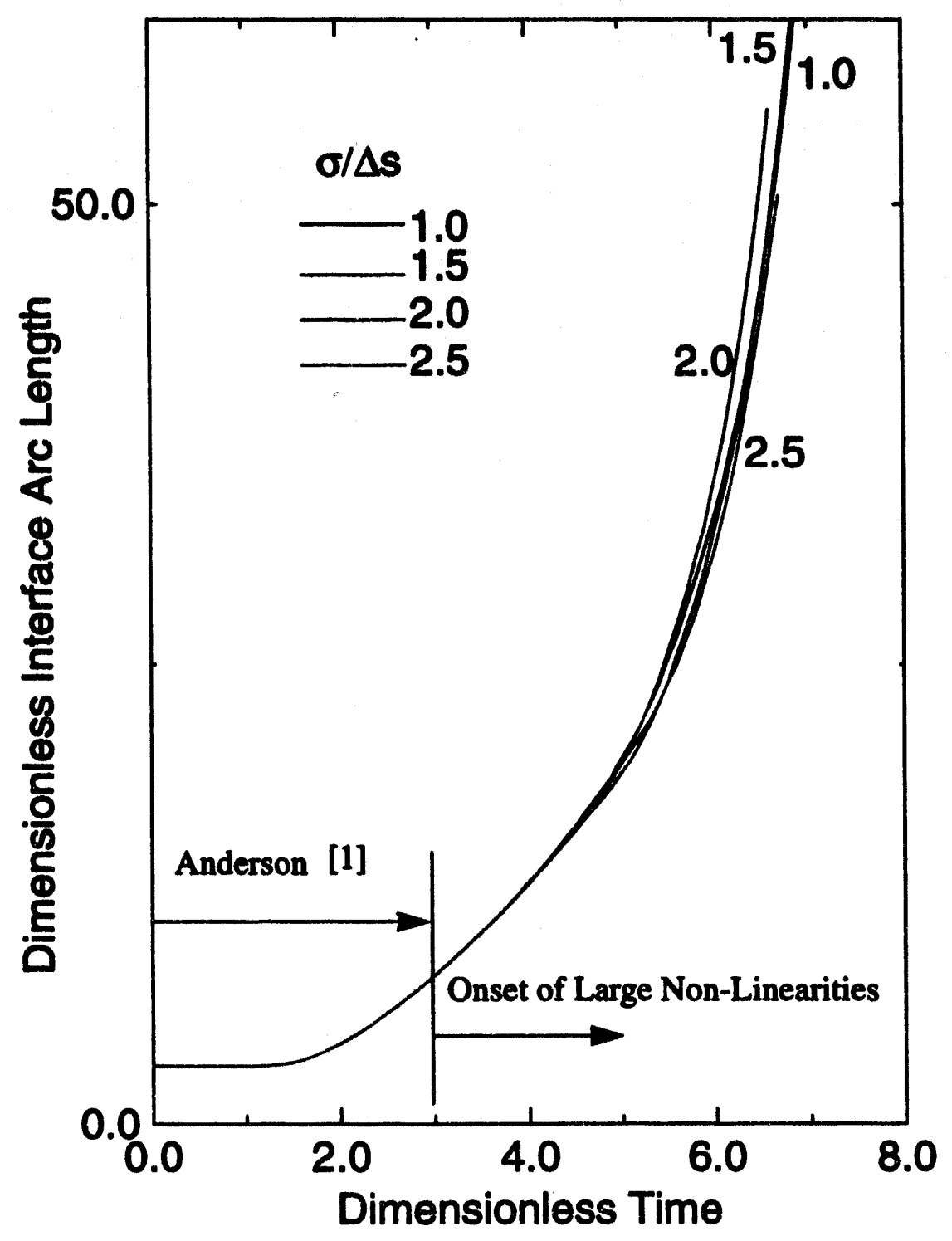

Figure 9 Increase in the length of the bubble interface versus time for solutions with adaption criteria of $\sigma / \Delta s=1.0,1.5,2.0,2.5$. The previous simulation of this problem by Anderson [1] was terminated at $t=3$, and is indistinguishable from the present solutions for that time interval. For $\mathrm{t}>3$, however, the interface length increases much more rapidly, as shown, which indicates the onset of large nonlinearities. Differences in the interface lengths for the different spacings are believed to be a manifestation of the inviscid equations which incessantly generate smaller length scales for smaller vortex spacings. 


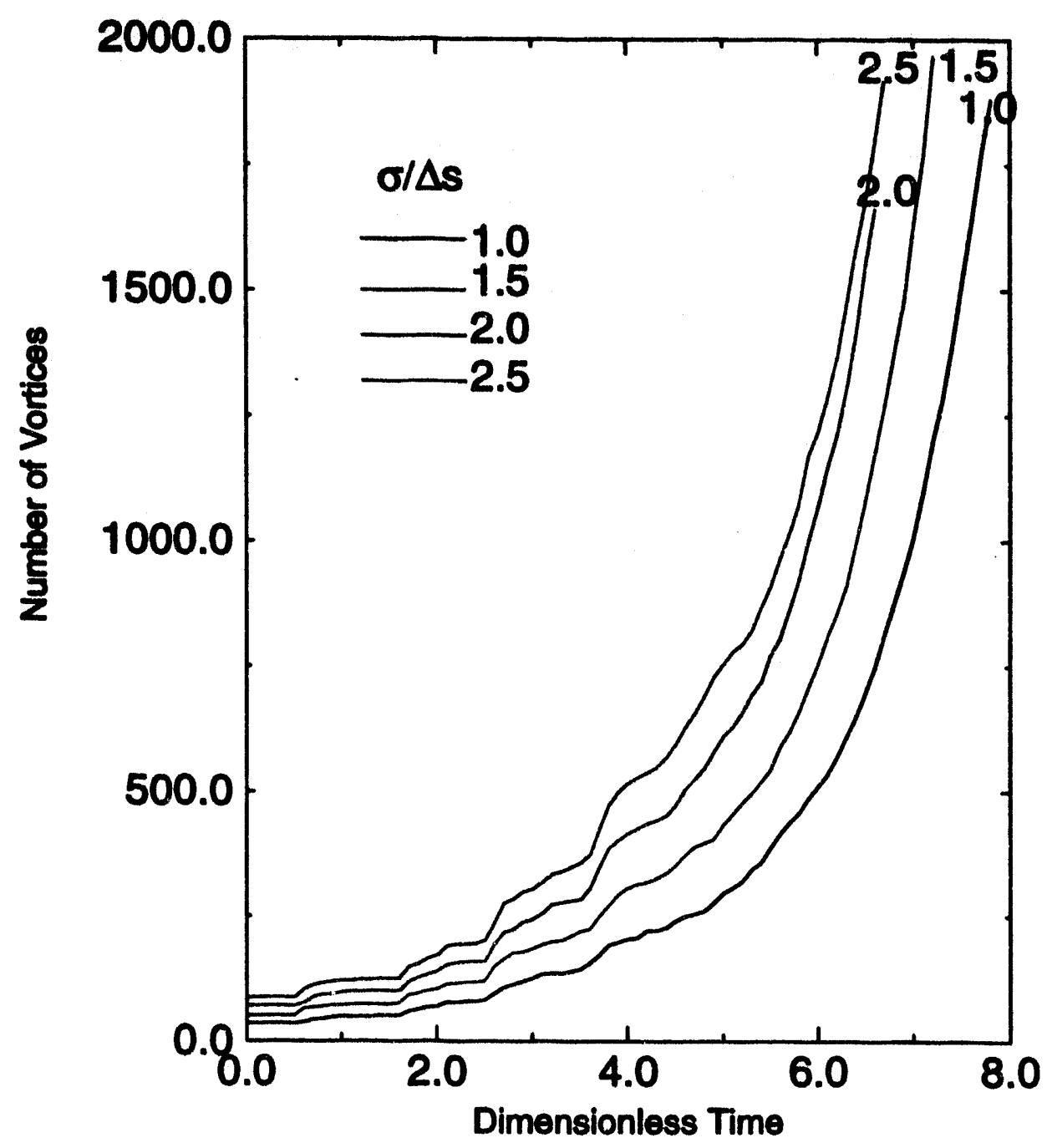

Figure 10 Increase in the number of vortices due to adaption for different adaption criteria.

From another point of view, the shape of the bubble at a dimensionless time of 6 is shown in Figure 11, for $\sigma / \Delta s=1,1.5,2.0$, and 2.5. Again, the shapes are similar, but clearly not the same. The result for $\sigma / \Delta s=0.5$ is also shown to emphasize the need to maintain $\sigma / \Delta s>$ 1 , as was found earlier to be a constraint to ensure smooth representation of the vorticity field.

One might hope that for vortex spacings less than some particular small value, the transient lengths and shapes would be independent ofvortex spacing. However, as mentioned earlier, the inviscid equations are believed to generate length scales as small as the numerical resolution allows. Thus, smaller scales are generated whenever the spacing between vortices becomes smaller. Thus, different solutions are obtained for different spacings because there is increased resolution for smaller vortex spacing, and as a result, "conver- 

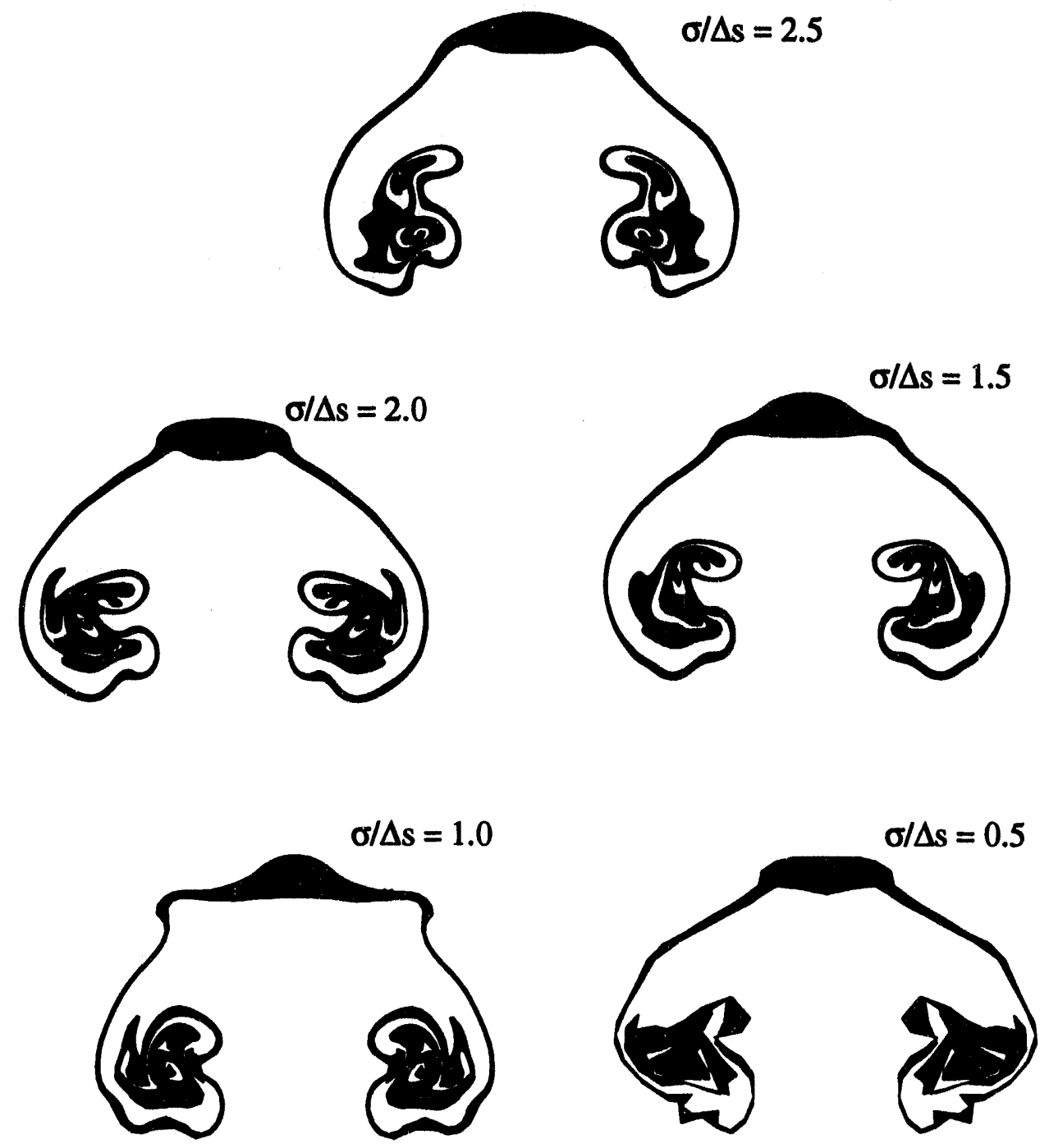

Figure 11 Bubble geometry at $t^{*}=6$ for different adaption criteria as shown. The smoothness of the shapes clearly increases as the distance between vortices decreases.

gence" in a general sense does not occur. Before addressing this issue further, we examine Anderson's previously published results for comparison with our solution.

Anderson's simulation [1] was terminated at a dimensionless time of 3 , and is reproduced in Figure 12. The number of vortices is constant for this calculation, so the adaption algorithm was not used, although the new formulation for the baroclinic term was used. This solution agrees in terms of the interface shape and length with the results published by Anderson, indicating that the new formulation of the baroclinic source term is appropriate. It is also noted that the adapted calculations discussed previously agree with the overall shape and transient length of the interface in Anderson's solution for $\sigma^{*}=0.1$. 


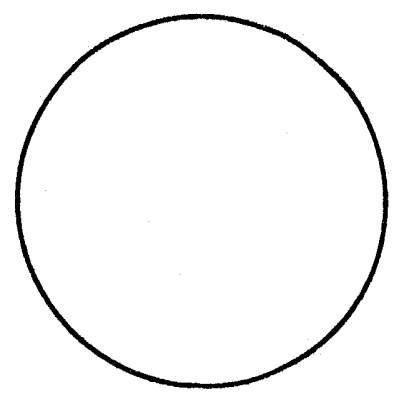

$t^{*}=0$
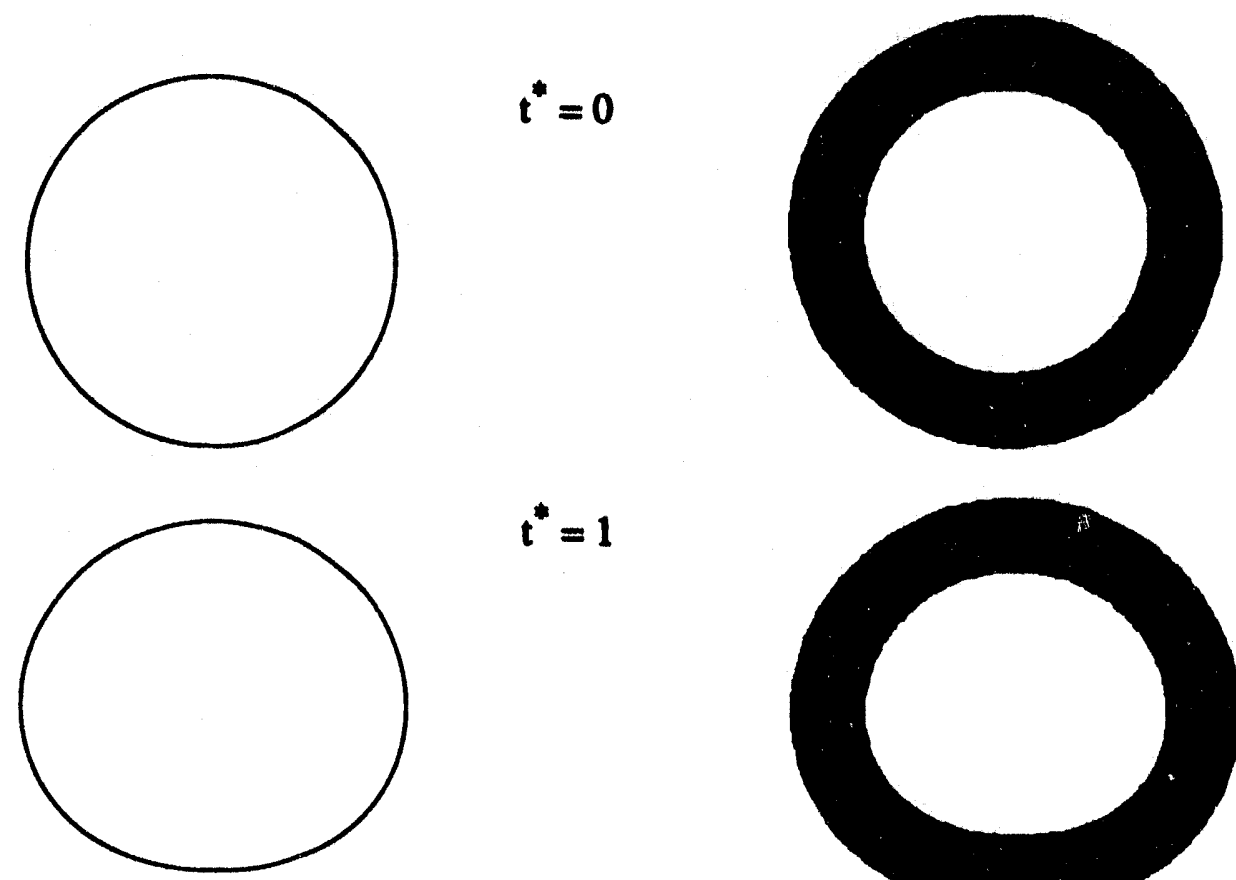

$t^{*}=1$
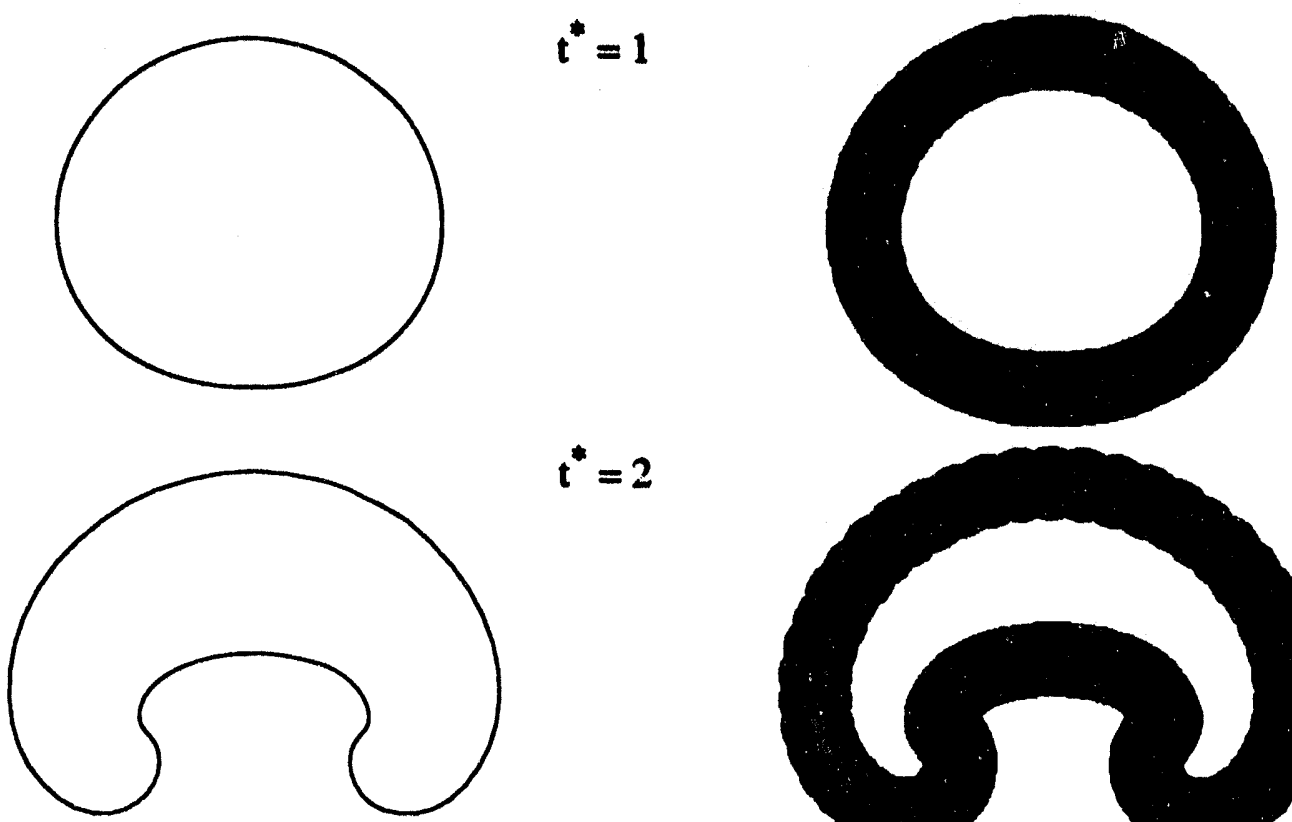

$t^{*}=2$
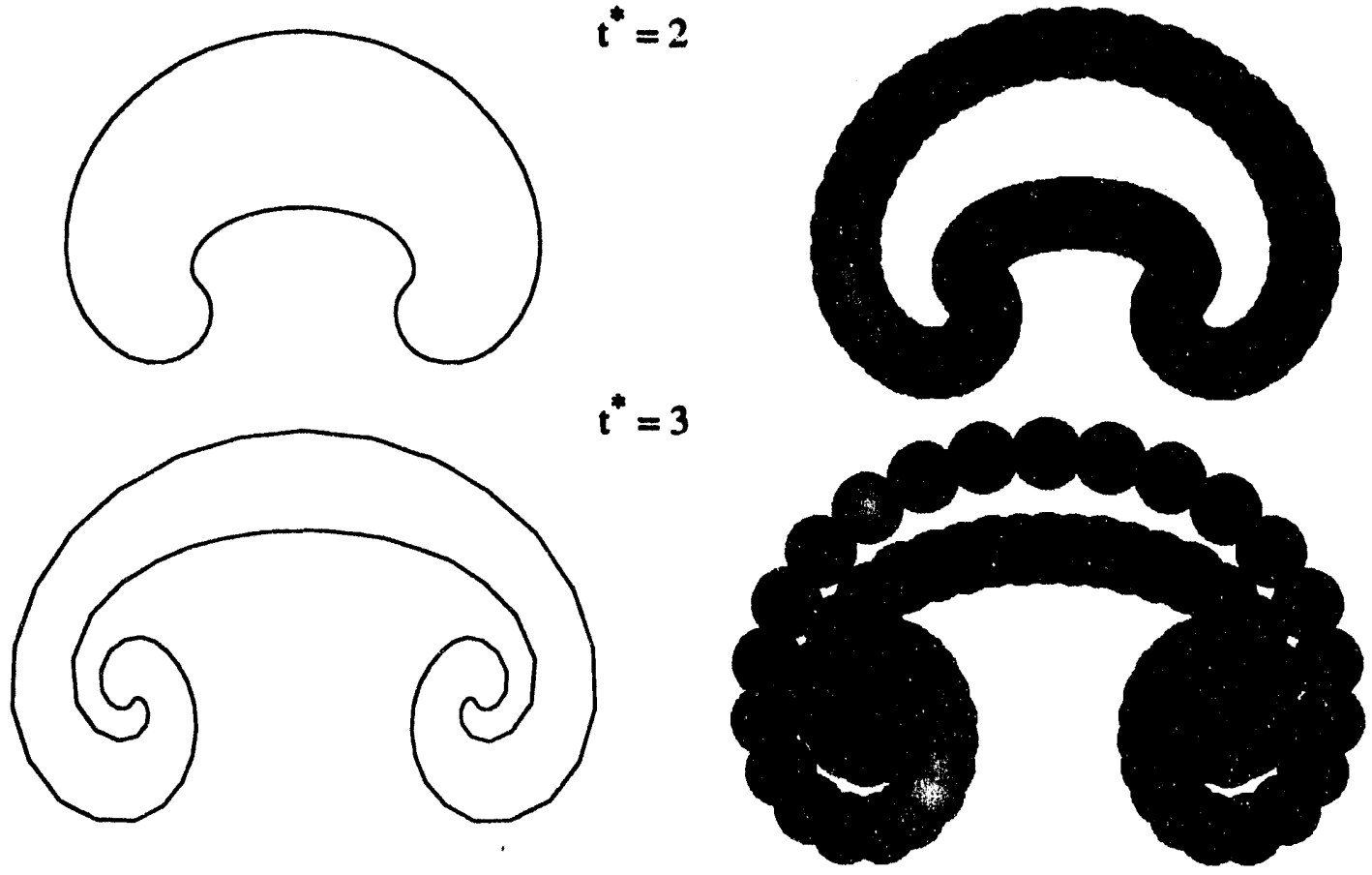

$-0.171$

Circulation

$+0.171$

Figure 12 Reproduction of calculations by Anderson [1] to simulate the evolution of a bubble using a fixed number of vortices. The lines formed by connecting the core centers indicate the interface shape, and the images. of the vortex cores allow the extent of core overlap to be examined. There is insufficient core overlap at $t^{*}=3$. The initial bubble radius is 1.0 , with core radii of 0.1 . 

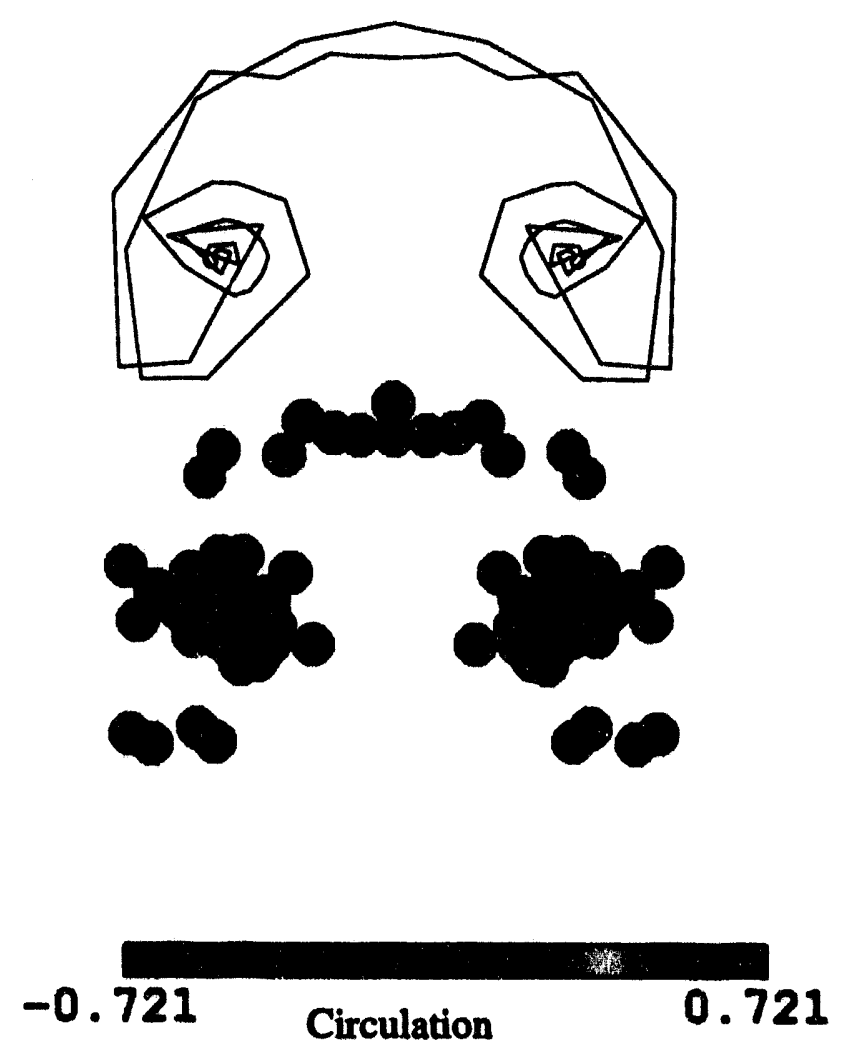

Figure 13 Cortinuation of solution in Figure 12 showing the consequences of failing to adapt the solution for late times: a non-physical solution at $\mathbf{t}=5$. The interface is shown to cross itself, which is nonphysical and is the result of the loss of core overlap.

These comparisons indicate that the new baroclinic formulation and the adaption algorithm provide accurate results, based on previous calculations, and allow the calculations to be carried out further than previous results.

To demonstrate the loss of accuracy that occurs if adaption is not performed, Anderson's solution was continued in time without adaption. Note that, in the reproduction of Anderson's solution, the vortices are initially overlapped considerably more than is necessary. This is to account for increasing separation between vortices as the interface stretches. In fact, the arc length of the interface increases by approximately 2.5 over the dimensionless time interval from 0 to 3 . To account for this, Anderson specified the initial core overlap to be $\sigma / \Delta s \approx 3$, or approximately 3 times more than the amount of overlap required for accuracy. Ostensibly, the factor of 3 is based on the finding that interface increased by approximately 2.5, plus a little more overlap for good measure. However, the interface stretches non-uniformly, so that at a time of 3 , some of the vortices, namely those at the top of the bubble, are no longer overlapped. As a result, continuing the solution past $t^{*}=3$ results in a catastrophic loss of accuracy, as shown in Figure 13, where the cores become further separated and the interface is no longer smooth. The use of our adaption technique prevents this loss of accuracy. 
a) $\sigma=0.05$

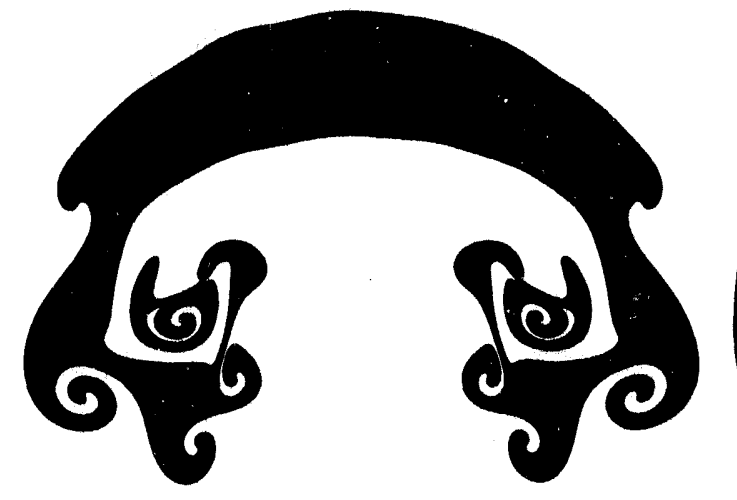

b) $\sigma=0.1$

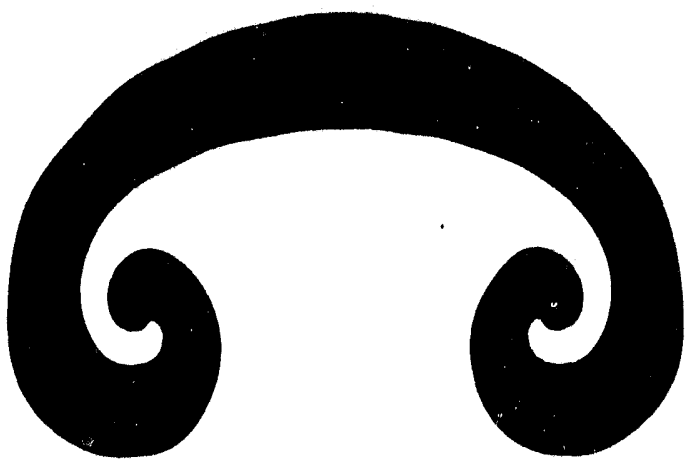

Figure 14 Bubble shapes at $\mathrm{t}^{*}=3$ for core radii of a) 0.05 and $\mathrm{b}$ ) 0.1 , with adaption criteria of 2.5. The solution using the smaller core size has many more small-scale features than the solution using the larger core size. Similar findings are also reported by Rottman and Stansby [20].

The effect of different core sizes is illustrated by simulating the bubble evolution with $\sigma=$ 0.05 (half the previous core size), and an adaption criteria of $\sigma / \Delta s=2.5$. The shape of the bubble at $\mathrm{t}^{*}=3$ is shown in Figure 14 for two core sizes: $\sigma=0.05$, and $\sigma=0.1$. Although the only difference between the two solutions is the size of the core, there are many more small-scale features in the solution for $\sigma=0.05$. This result is believed to be due to the generation of an entire spectrum of length scales by the inviscid Euler equations. The spectrum of length scales is believed to include infinitesimal length scales, which cannot be resolved in numerical solutions. As the resolution of a numerical solution increases, more and more small-scale features will be observed if the numerical method is not diffusive. Since vortex methods are not diffusive, this effect is clearly observed, whereas this would not be observed in methods that include artificial diffusion, since the diffusion would attenuate the small length scale features. Thus, in order to obtain a converged, realistic solution, the effects of real viscosity must be included. Nonetheless, the comparison of the two shapes in Figure 14 conveys the sense that a solution for a particular core size provides at least a macroscopic representation, so long as details of the small-scale structure are not of interest. 


\section{Summary}

A new vortex method was presented which allows the accurate simulation of inviscid, baroclinic flows. Two features of the method are new. First, a new formulation for the production of circulation is used which has the advantage that the gradient of the density field is not required; only the density itself is needed, thus eliminating one level of approximation in the model. To assess the accuracy of the new baroclinic formulation, the evolution of a bubble of lower density fluid was simulated and compared with a previously published simulation by Anderson. The overall shapes and lengths of the bubble interface were in good agreement, thus indicating that the new formulation is valid.

Second, a new adaption algorithm was formulated which conserves circulation, maintains monotonicity, and preserves symmetry. This adaption algorithm allows solutions to be performed to essentially arbitrary times without loss of core overlap, whereas previously results had to be terminated at relatively short times, since an effective adaption model was not available. The ability to simulate long times is desirable since many practical problems require long simulation times. Adapted short-time solutions of the bubble evolution were compared with previous solutions, and were in good agreement, indicating that the adaption did not introduce any new perturbations or inaccuracies. Continuation of these solutions to late times showed that the flow becomes highly nonlinear, as indicated by the complex shape of the bubble, and a rapid increase in the length of the bubble interface, which is indicative of chaotic mixing. Thus, the adaption algorithm allows simulations of mixing phenomena and internal wave phenomena at the times that are of interest for several applications beyond the naval applications that motivated this work.

Convergence of the solutions with respect to spacing of vortices and size of vortices was also considered, since it is important to be able to demonstrate convergence of numerical methods. The inviscid Euler equations are believed to generate a spectrum of lengthscales, including infinitesimal scales, which cannot be resolved using numerical methods, due to the fact that all numerical methods have finite resolution. Thus, in a sense, convergence of numerical solutions to the Euler equations is not a well-posed issue. Nonetheless, for simulations using a constant core size, solutions appear to converge as the spacing between vortices is reduced. For decreasing sizes of vortices (with fixed ratio of vortex spacing to core size), the shapes and interface length of the bubble varied considerably. This would not occur in simulations using many grid-based methods which require artificial viscosity, which attenuates small scale variations. Lagrangian vortex methods, on the other hand, do not require artificial viscosity, so the generation of small-scales is readily apparent. These findings provide further support for the view that the Euler equations generate infinitesimal scales.

The development of this inviscid model, in conjunction with the method to simulate viscous diffusion (also developed for this LDRD), provides a unique, accurate, gridless method to simulate finite Reynolds number flows. Efforts are underway to examine the convergence characteristics of the viscous, baroclinic model, where it is believed that converged solutions (with respect to core size and core spacing) can be readily obtained. After the convergence of this method is demonstrated, vortex methods will be closer than ever to being used to simulate flows for practical applications. 


\section{References}

1 Anderson, C. R., "A Vortex Method for Flows with Slight Density Variations," J. Comput. Phys., vol. 61, pp. 417-444, 1985.

2 Batchelor, G. K., An Introduction to Fluid Dynamics, Cambridge University Press, 1967.

3 Beale, J. T. and A. Majda, "Vortex Methods I: Convergence in Three Dimensions," Math. Comp., vol. 39, pp. 1-27, 1982.

4 Beale, J. T. and A. Majda, "Vortex Methods II: Higher Order Accuracy in Two and Three Dimensions," Math. Comput., vol. 39, pp. 29-52, 1982.

5 Brackbill, J. U., D. B. Kothe, and C. Zemach, "A Continuum Method for Modeling Surface Tension," J. Comput. Phys., vol. 100, pp. 335-354, 1992.

6 Collins, R., Chem. Eng. Sci., vol. 20, p. 851, 1965.

7 Chorin, A. J., "Numerical Study of Slightly Viscous Flow," J. Fluid. Mech., vol. 75, pp. 785-796, 1973.

8 Cottet, G. H. and S. Mas-Gallic, "A Particle Method to Solve the Navier-Stokes System," Numer. Math., vol. 57, pp. 805-827, 1990.

9 Dritschel, D. G., "Contour Surgery: A Topological Reconnection Scheme for Extended Integrations Using Contour Dynamics," J. Comput. Phys., vol. 77, pp. 240266.

10 Fishelov, D. “A New Vortex Scheme for Viscous Flows,” J. Comput. Phys., vol. 86, pp. 211-224, 1990.

11 Greengard, C., "The Core Spreading Vortex Method Approximates the Wrong Equation," J. Comput. Phys., vol. 61, pp. 345-347, 1985.

12 Ghoniem, A. F., G. Heidarninejad, and A. Krishanan, "Numerical Simulation of a Thermally Stratified Shear Layer Using the Vortex Element Method," J. Comp. Phys., vol. 79, pp. 135-166, 1988.

13 Hald, O., "The Convergence of Vortex Methods," SIAM J. Numer. Anal., vol. 16, pp. 725-755, 1979.

14 Kempka, S. N. and J. H. Strickland, "Simulation of the Viscous Transport of Vorticity by a Non-Solendoidal Diffusion Velocity," Sandia National Laboratories Report SAND93-1763, August, 1993. 
15 Meng, J. C. S. and J. A. L. Thompson, "Nurnerical Studies of Some Nonlinear Hydrodynamics Problems by Discrete Vortex Element Methods," J. Fluid Mech., vol. 84, pp. 433-453, 1978.

16 Ogami, Y. and T. Akamatsu, "Viscous Flow Simulation using the Discrete Vortex Model-The Diffusion Velocity Method," Computers \& Fluids, vol. 19, pp. 433-441, 1991.

17 Oran, J. S. and J. P. Boris, Numerical Simulation of Reactive Flow, Elsevier Publishing Co., 1987.

18 Puckett, E. G., "Vortex Methods: An Introduction and Survey of Selected Research Topics," Chapter 11 in Incompressible Computational Fluid Dynamics, Cambridge, 1993, Edited by M. D. Gunzburger and R. A. Nicolaides.

19 Russo, G., "A Deterministic Vortex Method for the Navier-Stokes Equations," J. Comput. Phys., vol. 108, pp. 84-94, 1993.

20 Rottman, J. W. and P. K. Stansby, "On the ' $\delta$-Equations' for Vortex Sheet Evolution," J. Fluid Mech., vol. 27, pp. 527-549, 1993.

21 Strickland, J. H. and S. N. Kempka, "Modeling Wake Evolution Behind a Submarine: Two-Dimensional Maneuvers," Sandia National Laboratories Report SAND930040, April 1993.

22 Tryggvason, G. "Vortex Dynamics of Stratified Flows," pp. 160-169 in Mathematical Aspects of Vortex Dynamics, SIAM, edited by Russel E. Caflisch, 1989.

23 Tryggvasson, G., "Numerical Simulations of the Rayleigh-Taylor Instability," J. Comput. Phys., vol. 75, pp. 253-282, 1988.

24 Wu, J. C. and J. Thompson, "Numerical Solutions of the Time-Dependent Incompressible Navier-Stokes Equations Using an Integro-Differential Formulation," Computers \& Fluids, vol. 1, pp. 197-215, 1973.

25 Zufiria, J. A., "Vortex-in-Cell Simulation of Bubble Competition in a Rayleigh-Taylor Instability," Phys. Fluids, vol. 31, no. 11, 1988. 


\section{Distribution}

Analytical Methods (2)

P.O. Box 3786

Dr. R. C. Maydew

Bellevue, WA 98009

5305 Queens Ct. NE

Attn: F. Dvorak

B. Maskew

Albuquerque, NM 87109

Prof. R. N. Meroney

Dept. of Civil Eng.

Mr. James Fein

Code 1221

ONR

800 N. Quincy St.

Arlington, VA 22217

Dr. H. Higuchi

Mech. Eng. \& Aero. Dept

Syracuse University

Syracuse, NY 13244

Capt. Hank Helin

HQ USFAVDFAN

USAF Academy, CO 80840-5701

Mr. Gary W. Jones

DARPA

Submarine Technology

1400 Wilson Blvd.

Arlington, VA 22209-2308

Prof. Joseph Katz

Dept. Aerospace Eng. and Eng. Mech.

San Diego State University

San Diego, CA 92115

Dr. George A. Keramidas

Code 4420

NRL

Washington, DC 20375

Prof. A. Leonard

Graduate Aeronautics Lab.

California Institute of Technology

Pasadena, CA 91125

Prof. M. Luttges

Dept. of Aerospace Eng. Sci.

Colorado State University

Fort Collins, CO 80521

NASA Johnson Space Center (2)

Attn: EG3

Houston, TX 77058

Attn: D. B. Kanipe

R. E. Meyerson

New Mexico State University (2)

Dept. of Mech. Eng.

Las Cruces, NM 88003

Attn: Ron Pederson

G. Reynolds

Mr. W. J. Quinlan

Aerodynamic Design

Ford Motor Company

21175 Oakwood Blvd.

Dearborn MI, 48123

Prof. T. Sarpkaya

Dept. Mech. Eng.

Code 69-SL

Naval Postgraduate Academy

Monterey, CA 93943

Prof. Roger L. Simpson

Dept. Aerospace and Ocean Eng.

Virginia Polytechnic Institute

and State University

Blacksburg, VA 24061

Mr. J. T. Strickland

Sterling Chemical Co.

Post Office Box 1311

Texas City, TX 77592

University of Colorado

Boulder, CO 80309-0429 
Texas Tech University (2)

Dept. of Mech. Eng.

Lubbock, TX 79409

Attn: J. H. Lawrence

J. W. Oler

University of New Mexico (2)

Dept. of Mech. Eng.

Albuquerque, NM 87106

Attn: M. S. Ingber

C. R. Truman

Dr. James Uhlman

Code 804, Bldg. 108/2

Naval Undersea Warfare Center

Newport, RI 02841-5047

$\begin{array}{lll}\text { MS 1351 } & 3020 & \text { W. R. Dawes } \\ \text { MS 0320 } & 1011 & \text { C. E. Meyers } \\ \text { MS 1111 } & 1421 & \text { W. J. Camp } \\ \text { MS 1111 } & 1421 & \text { D. W. Barnette } \\ \text { MS 0841 } & 1500 & \text { D. J. McCloskey } \\ \text { MS 0836 } & 1501 & \text { C. W. Peterson } \\ \text { MS 0827 } & 1502 & \text { P. J. Hommert } \\ \text { MS 0827 } & 1511 & \text { J. S. Rottler } \\ \text { MS 0827 } & 1511 & \text { D. K. Gartling } \\ \text { MS 0827 } & 1511 & \text { C. E. Hickox } \\ \text { MS 0827 } & 1511 & \text { S. N. Kempka (15) } \\ \text { MS 0834 } & 1512 & \text { A. C. Ratzel } \\ \text { MS 0834 } & 1512 & \text { M. R. Baer } \\ \text { MS 0834 } & 1512 & \text { J. R. Torczynski } \\ \text { MS 0835 } & 1513 & \text { R. D. Skocypec } \\ \text { MS 0835 } & 1513 & \text { S. Tiesczen } \\ \text { MS 0833 } & 1552 & \text { C. E. Hailey } \\ \text { MS 0833 } & 1552 & \text { R. S. Baty } \\ \text { MS 0833 } & 1552 & \text { J. H. Strickland (15) } \\ \text { MS 0826 } & 1553 & \text { W. L. Hermina } \\ \text { MS 0825 } & 1554 & \text { W. H. Rutledge } \\ \text { MS 0443 } & 1561 & \text { H. S. Morgan } \\ \text { MS 0437 } & 1562 & \text { R. K. Thomas } \\ \text { MS 0951 } & 2121 & \text { G. A. Laguna } \\ \text { MS 0511 } & 2500 & \text { G. N. Beeler } \\ \text { MS 0899 } & 7141 & \text { Technical Library (5) } \\ \text { MS 01007613-2Doc.Proc.DOE/OSTI (10) } \\ \text { MS 0619 } & 7151 & \text { Technical Publication (1) } \\ \text { MS 0149 } & 4000 & \text { J. C. Cummings } \\ \text { MS 0455 } & 4100 & \text { G. R. Otey } \\ \text { MS 0429 } & 5100 & \text { W. C. Nickell } \\ \text { MS 0574 } & 5941 & \text { D. F. Wolf } \\ \text { MS 0704 } & 6201 & \text { P. C. Klimas } \\ \text { MS 0708 } & 6214 & \text { H. M. Dodd } \\ \text { MS 0708 } & 6214 & \text { D. E. Berg } \\ & & \end{array}$

$\begin{array}{lll}\text { MS 9004 } & 8100 & \text { M. E. John } \\ \text { MS 9037 } & 8200 & \text { R. J. Detry } \\ \text { MS 9053 } & 8366 & \text { C. M. Hartwig } \\ \text { MS 9051 } & 8351 & \text { W. T. Ashurst } \\ \text { MS 9018 } & 8523-2 & \text { Central Tech Files } \\ \text { MS 0304 } & 9100 & \text { C. M. Hart } \\ \text { MS 0303 } & 9700 & \text { M. M. Newsom } \\ \text { MS 0860 } & 9122 & \text { E. G. Kadlec } \\ \text { MS 0411 } & 9723 & \text { J. M. Macha } \\ \text { MS 0860 } & 9122 & \text { R. L. Woodfin } \\ \text { MS 0105 } & 9835 & \text { R. B. Asher }\end{array}$




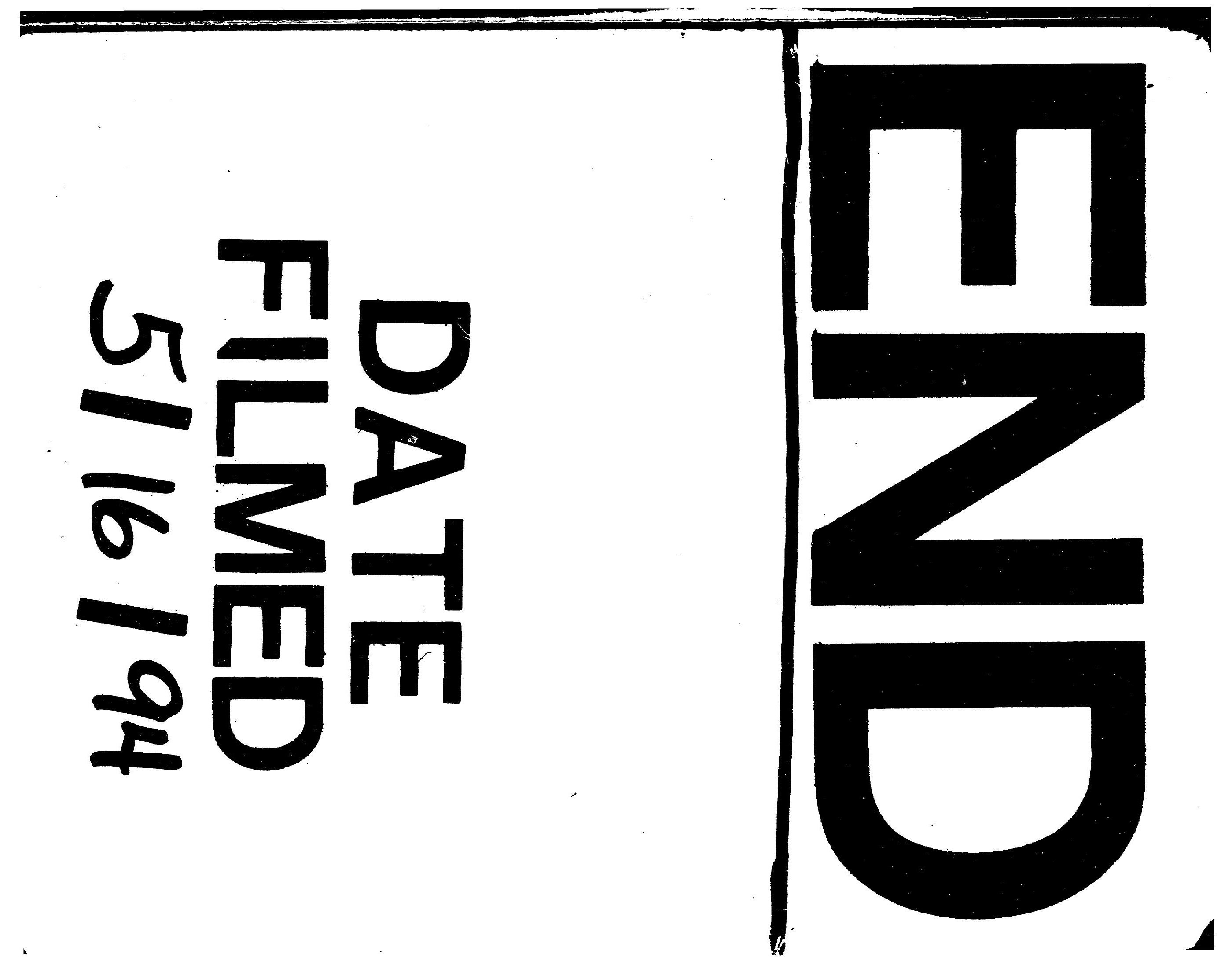

